AUS DEM LEHRSTUHL

FÜR EPIDEMIOLOGIE UND PRÄVENTIVMEDIZIN

PROF. DR. DR. MICHAEL LEITZMANN

DER FAKULTÄT FÜR MEDIZIN

DER UNIVERSITÄT REGENSBURG

SPECIFIC, BUT NOT GENERAL BELIEFS ABOUT MEDICINES ARE ASSOCIATED WITH MEDICATION ADHERENCE IN PATIENTS WITH COPD, BUT NOT ASTHMA: COHORT STUDY IN A POPULATION OF PEOPLE WITH CHRONIC PULMONARY DISEASE

\author{
Inaugural - Dissertation \\ zur Erlangung des Doktorgrades \\ der Medizin \\ der \\ Fakultät für Medizin \\ der Universität Regensburg
}

vorgelegt von

Wiebke Fischer 

AUS DEM LEHRSTUHL

FÜR EPIDEMIOLOGIE UND PRÄVENTIVMEDIZIN

PROF. DR. DR. MICHAEL LEITZMANN

DER FAKULTÄT FÜR MEDIZIN

DER UNIVERSITÄT REGENSBURG

SPECIFIC, BUT NOT GENERAL BELIEFS ABOUT MEDICINES ARE ASSOCIATED WITH MEDICATION ADHERENCE IN PATIENTS WITH COPD, BUT NOT ASTHMA: COHORT STUDY IN A POPULATION OF PEOPLE WITH CHRONIC PULMONARY DISEASE

\author{
Inaugural - Dissertation \\ zur Erlangung des Doktorgrades \\ der Medizin \\ der \\ Fakultät für Medizin \\ der Universität Regensburg
}

vorgelegt von

Wiebke Fischer 
Dekan:

1. Berichterstatter:

2. Berichterstatter:

Tag der mündlichen Prüfung:
Prof. Dr. Dr. Torsten E. Reichert

Prof. Dr. Christian Apfelbacher PhD

Prof. Dr. Michael Pfeifer

05. Oktober 2018 


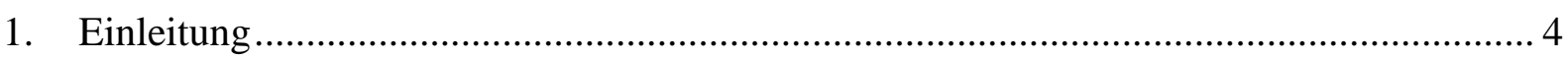

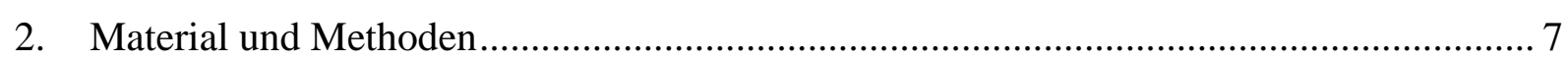

2.1. Studiendesign............................................................................................... 7

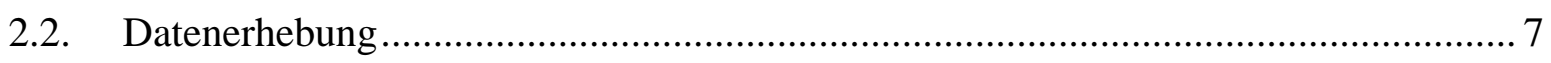

2.2.1. Abhängige Variable: Medikamentenadhärenz ................................................. 7

2.2.2. Unabhängige Variable: Medikamentenbezogene Überzeugungen ...................... 7

2.2.3. Potenzielle Confounder der Assoziation zwischen medikamentenbezogenen

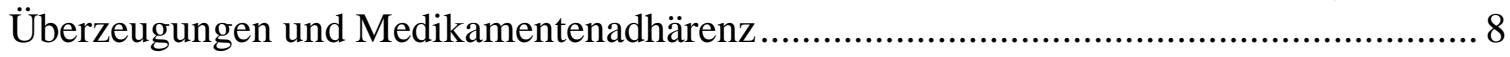

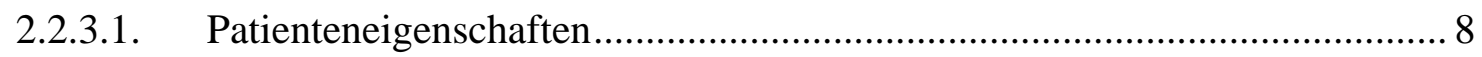

2.2.3.2. Krankheitsbezogene Faktoren.............................................................. 8

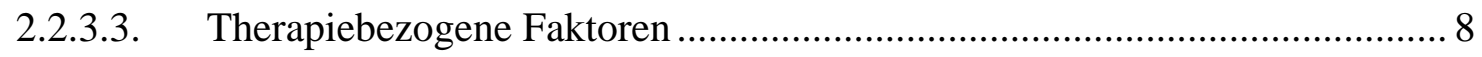

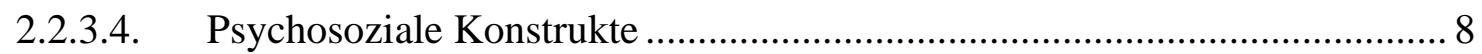

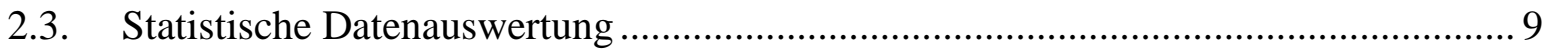

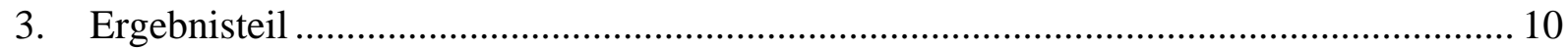

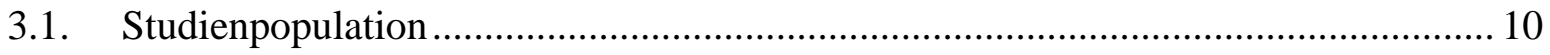

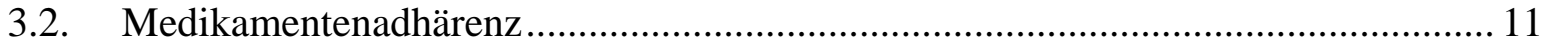

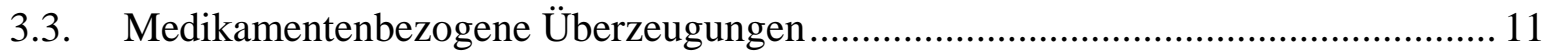

3.4. Assoziationen zwischen Medikamentenadhärenz und medikamentenbezogenen

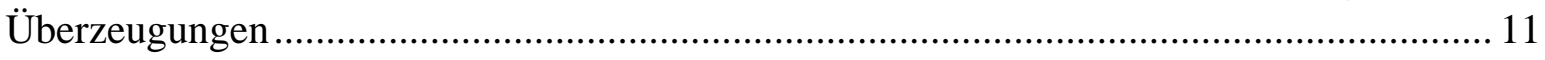

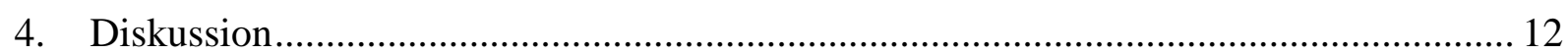

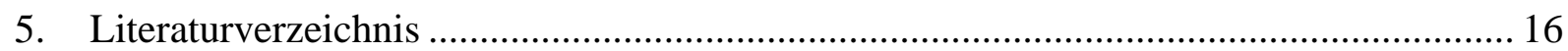

6. Eidesstattliche Erklärung

7. Danksagung

8. Lebenslauf

9. Originalartikel 


\section{Einleitung}

Asthma und die chronisch obstruktive Lungenerkrankung (COPD) sind chronische Lungenerkrankungen, welche sich durch eine Obstruktion der Atemwege auszeichnen, wobei diese bei Asthma reversibel (1), bei COPD hingegen konstant ist (2). Husten sowie Luftnot sind die Hauptsymptome beider Erkrankungen (2,3). Asthma und COPD zählen zu den häufigsten chronischen Erkrankungen (4). Asthma betrifft weltweit ca. 300 Millionen Menschen (5), die Prävalenz liegt in verschiedenen Ländern zwischen 1 und 18\% der Bevölkerung (1). Die COPD ist eine der führenden Todesursachen weltweit (6). Die Prävalenz der COPD liegt bei den über 40-Jährigen bei 5-10\% (3), ist beim männlichen Geschlecht höher (7) und steigt mit zunehmenden Alter (8) und der inhalierten Rauch-Dosis, welche in Packyears (dem Produkt aus der Anzahl der täglich konsumierten Zigarettenpackungen und der Rauchdauer in Jahren) angegeben wird (9). Bei Nichtrauchern tritt die COPD mit einer Prävalenz von 3-11\%, bei Rauchern mit einer Prävalenz von bis zu 25\% auf (8). Als chronische Erkrankungen stellen Asthma und COPD eine große Belastung des Gesundheitssystems dar (10). Die mittleren jährlichen Kosten für die Asthmabehandlung betragen in etwa $749 €$ pro Patient (11). Die Kosten für ein schlecht eingestelltes Asthma sind in etwa doppelt so hoch wie die eines gut eingestellten Asthmas (12). Für die COPD liegen die direkten jährlichen Kosten (durch Therapien, Krankenhausaufenthalte) pro Patient in Deutschland zwischen $4441 €$ (GOLD St. I) und $10.770 €$ (GOLD St. IV), die indirekten Kosten (für Arbeitsausfälle, Frühberentung) bei $14.758 €($ GOLD St. I) bis $33.795 €$ (GOLD St. IV) jährlich (13).

$\mathrm{Zu}$ den Therapiemassnahmen beider Erkrankungen zählen medikamentöse (Kortikosteroide, Bronchodilatatoren) und nicht medikamentöse Strategien (z.B. Rauchentwöhnung) $(1,2)$. Durch eine optimale Therapie kann der Gesundheitszustand beider Patientengruppen deutlich verbessert (14), das Risiko von Exazerbationen vermindert (15) und die Zahl der Hospitalisierungen aufgrund der Erkrankung reduziert werden (16). Für eine optimale Therapie ist es wichtig, dass die Patienten die Medikamente regelmäßig und wie verordnet einnehmen. Nichtsdestotrotz haben Studien gezeigt, dass die Adhärenz bei Patienten mit chronischen Lungenerkrankungen wie Asthma und COPD niedrig ist (17,18), z.T. liegt sie bei nur 40-70\% $(19,20,21,22)$. Adhärenz beschreibt das Ausmaß, in dem der Patient dem mit dem behandelnden Arzt gemeinschaftlich erarbeiteten Therapieplan folgt (23). Verglichen mit dem häufig verwendeten Begriff der Compliance, betont der Begriff Adhärenz die aktive Rolle des Patienten unter Anleitung des Arztes (24). 
Um die Therapieadhärenz der Patienten verbessern zu können, ist es primär wichtig, Adhärenz beeinflussende Faktoren zu untersuchen. Die Weltgesundheitsorganisation (WHO) hat fünf Dimensionen identifiziert, welche die Adhärenz nennenswert beeinflussen: Sozioökonomische Faktoren, therapiebezogene Faktoren, patientenbezogene Faktoren, krankheitsbezogene Faktoren und Faktoren, die mit dem Gesundheitssystem zusammenhängen (23). Studien zeigen, dass soziodemographische Faktoren (wie z.B. das Alter, das Geschlecht, der Bildungsstand), krankheitsbezogene Faktoren (wie die Erkrankungsdauer) und auch therapiebezogene Faktoren (wie die Zahl der Hospitalisierungen) nur einen geringen oder unklaren Einfluss auf das Adhärenzverhalten haben $(20,25,26)$. Hingegen sind patientenbezogene Faktoren wie die krankheitsbezogene Wahrnehmung und die Überzeugungen, die Patienten im Hinblick auf ihre Medikamente besitzen in den Fokus der Adhärenzforschung gerückt (27). Diese Überzeugungen werden auch als ,beliefs about medicines“ bezeichnet und wurden von Horne in fünf Domänen eingeteilt: Allgemein-Schädlichkeit, Allgemein-Nützlichkeit, Allgemeinübermäßiger Gebrauch, Spezifisch-Notwendigkeit und Spezifisch-Befürchtungen (27,28). Die ersten drei Domänen spiegeln die allgemeinen Ansichten der Patienten im Hinblick auf Medikamente wider, so zum Beispiel, dass alle Medikamente chemisch hergestellte Produkte und damit potenziell giftig sind (Allgemein-Schädlichkeit), dass Medikamente vielen Menschen helfen, trotz ihrer Erkrankung ein beschwerdefreies Leben zu führen (Allgemein-Nützlichkeit) oder dass Ärzte Medikamente anderen Therapieoptionen zu häufig vorziehen (Allgemeinübermäßiger Gebrauch). Die letzten beiden Domänen repräsentieren spezifische Ansichten: Zum einen, dass die eigenen Medikamente eine Progression der Erkrankung verlangsamen und Symptome abschwächen (Spezifisch-Notwendigkeit). Zum anderen, dass sie Nebenwirkungen verursachen und abhängig machen (Spezifisch-Befürchtungen) (28,29). Die „beliefs about medicines" wurden aus verschiedenen konzeptuellen Modellen entwickelt. Eines dieser Modelle ist das so genannte Common Sense Model (CSM), welches beschreibt, dass Patienten vorwiegend mit zwei Aspekten in Bezug auf ihre Krankheitswahrnehmung konfrontiert werden: zum einen mit der durch die Krankheit empfundenen Bedrohung (kognitives Niveau), zum anderen mit den dadurch ausgelösten Emotionen (emotionales Niveau). Die individuelle Verarbeitung dieser Aspekte wird durch medikamentenbezogene Überzeugungen beeinflusst (30). Ob nach dem Common Sense Model bei einer wahrgenommenen Bedrohung durch die Erkrankung eine Medikamenteneinnahme erfolgt, hängt von der individuellen Bewertung sowie von persönlichen Erfahrungen ab. In die Bewertung fließt neben der Überzeugung der Patienten, dass sie eine Therapie benötigen auch die Angst vor möglichen Nebenwirkungen ein. 
Das Modell des Necessity-Concerns Frameworks, welches auf Grundlage des CSM entwickelt wurde, beschreibt das individuelle Abwägen hinsichtlich einer Medikamenteneinnahme (31). Medikamentenbezogene Überzeugungen wurden in verschiedenen Studien erfasst, um Adhärenz bei chronisch-kranken Patienten vorherzusagen $(25,32)$.

Bei Patienten mit Asthma waren Befürchtungen im Hinblick auf ihre Medikamente ebenso wie Überzeugungen, dass Medikamente generell schädlich sind und zu häufig verwendet werden, mit einer verringerten Adhärenz verknüpft $(25,26,33)$. Andere Studien zeigten eine positive Assoziation zwischen der Domäne Spezifisch-Notwendigkeit und Adhärenz bei Patienten mit Asthma $(22,34,35)$, sowie ein höheres Risiko der Non-Adhärenz bei Patienten, welche eher besorgt waren im Hinblick auf ihre Medikation (21). Für Patienten mit COPD wurde der Zusammenhang zwischen medikamentenbezogenen Überzeugungen und Adhärenz bisher in nur wenigen Studien untersucht (20,36,37). Die Ergebnisse dieser Studien stützen weitestgehend die Ergebnisse der Untersuchungen bei Patienten mit Asthma.

Ein besseres Verständnis des Einflusses von medikamentenbezogenen Überzeugungen auf das Adhärenzverhalten bei Patienten mit Asthma und COPD könnte ein essentieller Schritt hinsichtlich der Verbesserung der Medikamentenadhärenz und konsekutiv der Reduktion von Krankheitskosten sowie der Verbesserung des Gesundheitszustands bedeuten (23).

In der Literatur findet sich nur eine prospektive Studie aus Australien, welche Einflussfaktoren von Adhärenz bei Patienten mit Asthma untersuchte (38). Für Patienten mit COPD konnten wir keine prospektive Studie finden. Nichtsdestotrotz werden prospektive Studien benötigt, um einen möglichen kausalen Zusammenhang der Assoziation von medikamentenbezogenen Überzeugungen und Adhärenz zu untersuchen. Das Ziel unserer Studie war es, die Beziehung dieser Überzeugungen und dem Adhärenzverhalten bei Patienten mit COPD und Asthma über einen längeren Zeitraum zu untersuchen. Insbesondere wurden die Hypothesen untersucht, dass die Punktwerte der BMQ Subskalen Spezifisch-Befürchtungen, Allgemein-übermäßiger Gebrauch und Allgemein-Schädlichkeit invers und die Punktwerte der Subskalen Allgemein-Nützlichkeit und Spezifisch-Notwendigkeit positiv mit Adhärenz assoziiert sind. 


\section{Material und Methoden}

\subsection{Studiendesign}

Für die Erhebung der Baselinedaten wurden Patienten im Zeitraum Juni 2013 bis Dezember 2014 in Krankenhäusern und ambulanten Hausarzt- und Facharztpraxen in der Umgebung von Regensburg, Deutschland rekrutiert. Eingeschlossen wurden volljährige Patienten mit der Hauptdiagnose Asthma oder COPD und einer Mindestkrankheitsdauer von 3 Monaten. Patienten mit akuten psychiatrischen Erkrankungen (mit Ausnahme von Depressionen und Angststörungen) wurden ausgeschlossen. Nach der schriftlichen Einwilligung zur Teilnahme wurden die Daten mittels Fragebogen und durch Einsichtnahme in die Krankenakte der Patienten erhoben. Die Follow-Up Erhebungen wurden nach drei Monaten (von September 2013 bis März 2015) und nach einem Jahr (von Juni 2014 bis Dezember 2015) per Postversand der Fragebögen durchgeführt. Reminderanschreiben und Erinnerungsanrufe zielten auf eine möglichst große Studienpopulation über alle drei Messzeitpunkte ab.

\subsection{Datenerhebung}

\subsubsection{Abhängige Variable: Medikamentenadhärenz}

Die Medikamentenadhärenz wurde zu allen drei Messzeitpunkten mit der deutschen Version des Medication Adherence Report Scale (MARS) Fragebogens erhoben, welcher als geeigneter Fragebogen zur Detektion von Non-Adhärenz beschrieben wurde (39) und aus fünf Frageitems besteht. Der gebildete Summenscore liegt zwischen 5 und 25, in unsere Studie galt ein Score von 25 als adhärent, ein Score <25 als non-adhärent. Es wurden Sensitivitätsanalysen mit verschiedenen weiteren Cut-off-Werten durchgeführt.

\subsubsection{Unabhängige Variable: Medikamentenbezogene Überzeugungen}

Zur Messung wurde der Beliefs about Medicines Questionnaire (BMQ) (29,40) verwendet. Dieser besteht aus 23 Items auf den folgenden fünf Skalen: Spezifisch-Notwendigkeit, Spezifisch-Befürchtungen, Allgemein-Schädlichkeit, Allgemein-Nützlichkeit und Allgemeinübermäßiger Gebrauch. Je höher der Skalenwert, desto ausgeprägter sind die Überzeugungen in der jeweiligen Domäne. 
2.2.3. Potenzielle Confounder der Assoziation zwischen medikamentenbezogenen Überzeugungen und Medikamentenadhärenz

\subsubsection{Patienteneigenschaften}

Soziodemographische Daten wie Alter, Geschlecht, Bildungsstand und Lebenssituation wurden für alle Patienten erhoben. Der Bildungsstand wurde kategorisiert: niedriger Bildungsstand bei Schulbildung < 10 Jahren, mittlerer Bildungsstand bei 10 Jahren Schulbildung und hoher Bildungsstand bei mehr als 10 Jahren Schulbildung. Bei der Baselineerhebung wurden nur die Patienten mit COPD nach ihrem Rauchverhalten gefragt.

\subsubsection{Krankheitsbezogene Faktoren}

Krankheitsbezogene Daten wie Zeitraum seit Diagnosestellung, Anzahl der Komorbiditäten sowie Angaben über die Zahl der Exazerbationen in den letzten Monaten und über Nebenwirkungen durch Medikamente wurden erhoben. Der Schweregrad der Erkrankung wurde mittels der Einsekundenkapazität ( $\left.\mathrm{FEV}_{1}\right)(2)$, dem COPD Assessment Test (CAT) (41) und dem Asthma Control Questionnaire (ACQ) (42) erhoben. Der Schweregrad der COPD wurde anhand der Global Initiative for Chronic Obstructive Lung Disease (GOLD) Kategorien eingeteilt (2). Der CAT ist ein Fragebogen mit acht Items, welche die typischen Symptome und Einschränkungen aufgrund der COPD Erkrankung erfassen (41). Ein höherer Summenscore bedeutet größere Einschränkungen im Alltag durch die COPD Erkrankung (43). Der $A C Q$ Fragebogen besteht aus sieben Items und erfasst Symptome, Exazerbationen und den Gebrauch von Reliever-Medikamenten. Ein hoher Mittelwert beschreibt mehr Einschränkungen der Patienten durch die Erkrankung und ein schlecht kontrolliertes Asthma.

\subsubsection{Therapiebezogene Faktoren}

Die Entfernungszeit zum Behandler in Minuten, die Anzahl der Arztbesuche in den letzten Monaten, die Anzahl der Medikamente (Inhalativa, Tabletten, Andere) und die Angaben über eine ambulante oder stationäre Behandlung wurden für alle Patienten erhoben.

\subsubsection{Psychosoziale Konstrukte}

- Generische Lebensqualität

Die Lebensqualität der Patienten wird durch Erkrankungen wie Asthma und COPD deutlich eingeschränkt $(44,45)$. In unserer Studie wurde die Lebensqualität mittels des Short-Form Health Questionnaire 12 (SF-12) erfasst, welcher zwei Skalen für körperliche (Physical 
Component Scale) und psychische Faktoren (Mental Component Scale) beinhaltet. Höhere Scores bedeuten auch eine höhere Lebensqualität (46).

- Allgemeine Selbstwirksamkeit

Die allgemeine Selbstwirksamkeit wurde mittels der General Self Efficacy Scale (GSE) erfasst, welche 10 Frageitems beinhaltet. Der Gesamtscore liegt zwischen 10 und 40 Punkten, wobei eine höhere Punktzahl eine höhere Selbstwirksamkeit bedeutet (47).

- Depressionen und Angststörungen

Depressive Komorbidität und Angststörungen sind nicht selten bei Patienten mit Asthma und COPD. Bis zu ein Drittel der Patienten mit Asthma, vor allem Frauen, leidet unter Depressionen $(48,49)$. Schätzungsweise $8-80 \%$ aller Patienten mit COPD sind an einer Depression erkrankt (50). Jeder fünfte junge Patient mit Asthma leidet zudem unter Angststörungen (48), bei den Patienten mit einer COPD liegt die Prävalenz bei 6-74\%, je nach diagnostischem Vorgehen (50). Depression ist ein Risikofaktor für Non-Adhärenz $(51,52)$, während der Einfluss von Angststörungen auf das Adhärenzverhalten variabel und häufig nicht signifikant ist (51). In unserer Studie wurde die Hospital Anxiety and Depression Scale (HADS) (53) zur Messung dieser Komorbiditäten verwendet. Der Gesamtscore wird mittels einer Depressionssubskala und einer Angstsubskala erhoben und liegt zwischen 0 und 21 Punkten, wobei eine höhere Punktzahl eine höhere Wahrscheinlichkeit einer begleitenden Depression oder Angststörung darstellt.

\subsection{Statistische Datenauswertung}

Die Datenauswertung wurde für beide Patientengruppen getrennt mit IBM SPSS Statistics 24.0 durchgeführt. Fehlende Patientendaten führten nicht zu einem generellen Ausschluss dieser Patienten, sondern lediglich zu einem Ausschluss der Patienten von den entsprechenden Analysen. Das Signifikanzniveau wurde auf 0.05 gesetzt. Mittels Mann-Whitney U und Fisher's Exact Tests wurden die Charakteristika der Patienten, welche bei der letzten FollowUp Befragung teilnahmen, mit denen verglichen, die die Studie vorzeitig abbrachen, um mögliche Risikofaktoren des sogenannten Loss to Follow-Up zu identifizieren. Für jede Follow-Up Erhebung und jede Patientengruppe wurde eine multivariable logistische Regressionsanalyse mit den BMQ Subskalenwerten der Baseline als unabhängige Variable und der Adhärenz (zu den verschiedenen Zeitpunkten) als dichotomisierte, abhängige Variable 
durchgeführt. Univariate Analysen wurden zur Identifikation möglicher Confounder der Assoziation zwischen Adhärenz und medikamentenbezogenen Überzeugungen durchgeführt. $\mathrm{Zu}$ diesen möglichen Confoundern zählten patienten-, therapie- und krankheitsbezogene Faktoren genauso wie psychosoziale Konstrukte, welche im Rahmen der Baseline erhoben wurden. Bei einer Assoziation dieser Faktoren mit der Adhärenz $(\mathrm{p} \leq 0.20)$ wurden diese ins multivariable Modell eingeschlossen. Alle Variablen wurden auf Multikollinearität getestet. Um die Modelle vergleichbar zu machen, wurden nur Variablen in die multivariable Analyse eingeschlossen, die bei der Baselineerhebung für beide Patientengruppen erhoben wurden. Abschließend wurde eine Sensitivitätsanalyse des finalen Modells mit nur den Patienten durchgeführt, die an der letzten Follow-Up Befragung teilnahmen.

\section{Ergebnisteil}

\subsection{Studienpopulation}

Insgesamt nahmen 402 Patienten an der Studie teil, von denen ca. die Hälfte an Asthma erkrankt war. Knapp die Hälfte der Teilnehmer waren Frauen, das Durchschnittsalter betrug 56.7 Jahre (SD 15.9). Der Großteil der Patienten (84.2\%) hatte einen niedrigen oder mittleren Bildungsstand. Über $60 \%$ der Patienten waren in ambulanter Behandlung. Von den Patienten mit Asthma waren 95.4\%, von den Patienten mit COPD 30.6\% ambulant betreut. Knapp 2/3 der Patienten mit COPD waren dem Stadium GOLD II (32.2\%) und GOLD III (33.6\%) zuzuordnen. 28\% von ihnen hatten eine COPD des Schweregrades GOLD IV, während 6.3\% dem Stadium GOLD I entsprachen. Der Mittelwert des CAT Scores betrug 23.3 (SD 7.2). Ein mittlerer ACQ Score von 1.5 (SD 1.2) sprach für ein weitestgehend gut kontrolliertes Asthma unter den Probanden.

An der Follow-Up Befragung nach drei Monaten nahmen noch 255 Patienten teil, von denen knapp die Hälfte Frauen waren (49.4\%) und 43.5\% Asthma hatten. Nach einem Jahr verblieben 171 Patienten in der Studie. Davon hatten $42.7 \%$ eine Asthmaerkrankung und $49.7 \%$ waren weiblich. Die Eigenschaften der Patienten, welche an der gesamten Studie teilnahmen, wiesen zwei signifikante Unterschiede im Vergleich zu den Patienten auf, welche frühzeitig die Studie beendeten: Zum einen waren die Patienten, die weiterhin teilnahmen älter $(M d n=62.3$, $Q 1 / Q 3=53.8 / 67.7 ; M d n=57.7, Q 1 / Q 3=41.5 / 67.6, U=22.453, p=.019)$, zum anderen hatten sie ihren Arzt in den letzten 12 Monaten häufiger besucht $(M d n=4, Q 1 / Q 3=2 / 6 ; M d n=3$, 
$Q 1 / Q 3=2 / 5, U=21.151, p=.007)$. Bezüglich des Adhärenzverhaltens zur Baselineerhebung zeigte sich kein signifikanter Unterschied zwischen Patienten, die weiterhin an der Studie teilnahmen und denen, die diese frühzeitig beendeten.

\subsection{Medikamentenadhärenz}

Nach drei Monaten gaben 30.6\% der Patienten an, adhärent zu sein. Bei den Patienten mit COPD lag der Wert bei 43\%, bei denen mit Asthma bei 14.5\%. Nach einem Jahr gab noch gut ein Drittel der Patienten (33.9\%) ein adhärentes Verhalten an. Bei den Patienten mit Asthma waren es nach einem Jahr 17.8\%, die sich als adhärent beschrieben, bei den Patienten mit COPD $45.9 \%$.

\subsection{Medikamentenbezogene Überzeugungen}

Zum Zeitpunkt der Baselineerhebung waren die Scores der BMQ Subskalen AllgemeinNützlichkeit $(\mathrm{M}=3.9, \mathrm{SD}=0.68)$ und Spezifisch-Notwendigkeit $(\mathrm{M}=3.9, \mathrm{SD}=0.90)$ besonders hoch. Niedrig waren sie hingegen für die Subskala Spezifisch-Befürchtungen $(\mathrm{M}=2.4$, $\mathrm{SD}=0.78$ ). Patienten, welche an der Follow-Up-Erhebung nach einem Jahr teilnahmen, hatten einen signifikant höheren Spezifisch-Notwendigkeit Score $(M d n=4.2, \quad Q 1 / Q 3=3.4 / 4.7)$ gegenüber Patienten, die frühzeitig die Studie beendeten $(M d n=3.8, Q 1 / Q 3=3.2 / 4.55$, $U=22.195, p=0.009)$. Für die anderen BMQ Subskalen konnten keine signifikanten Unterschiede identifiziert werden.

\subsection{Assoziationen zwischen Medikamentenadhärenz und medikamentenbezogenen} Überzeugungen

Bei der Auswertung der Baselinedaten zeigten sich signifikante Assoziationen zwischen den allgemeinen medikamentenbezogenen Überzeugungen und Adhärenz: Allgemein-Schädlichkeit und Allgemein-übermäßiger Gebrauch waren signifikant invers assoziiert mit Medikamentenadhärenz. Des Weiteren zeigte sich eine positive signifikante Assoziation zwischen Spezifisch-Notwendigkeit und Adhärenz bei beiden Patientengruppen. Die Ergebnisse der Baselineauswertung wurden bereits publiziert (54). Bei der Analyse der prospektiven Daten zeigte sich, dass ein höherer Score auf der Subskala Allgemein-übermässiger Gebrauch für Patienten mit Asthma sowohl bei der Erhebung nach 3 Monaten $(\mathrm{OR}=0.76, \mathrm{p}=0.483)$ als auch nach einem Jahr $(\mathrm{OR}=0.42$, $\mathrm{p}=0.094)$ mit einer geringeren Chance auf adhärentes Verhalten verknüpft war. Bei den Patienten mit COPD war Allgemein-übermässiger Gebrauch ebenfalls mit niedrigeren Chancen auf adhärentes Verhalten nach 3 Monaten $(\mathrm{OR}=0.77, \mathrm{p}=0.328)$ und 
nach einem Jahr $(\mathrm{OR}=0.60, \mathrm{p}=0.125)$ assoziiert. Allgemein-Schädlichkeit verringerte bei Patienten mit Asthma, die Chancen auf Medikamentenadhärenz sowohl nach 3 Monaten $(\mathrm{OR}=0.75, \mathrm{p}=0.549)$ als auch nach einem Jahr $(\mathrm{OR}=0.33, \mathrm{p}=0.084)$. Für die Patienten mit COPD konnte ebenfalls eine inverse Assoziation zwischen Allgemein-Schädlichkeit und Adhärenz nach 3 Monaten $(\mathrm{OR}=0.91, \mathrm{p}=0.738)$ und einem Jahr gezeigt werden $(\mathrm{OR}=0.67$, $\mathrm{p}=0.293$ ). Höhere Scores auf der Subskala Allgemein-Nützlichkeit waren generell mit einer höheren Chance auf adhärentes Verhalten bei beiden Patientengruppen verbunden. Die einzige Ausnahme bildete die Gruppe mit COPD bei der Erhebung nach einem Jahr $(\mathrm{OR}=0.93$, $\mathrm{p}=0.850$ ). Keine der Assoziationen zwischen den allgemeinen medikamentenbezogenen Überzeugungen und Medikamentenadhärenz erreichte das Signifikanzniveau. Für die Patienten mit Asthma zeigte sich eine positive Assoziation zwischen höheren Scores auf der Subskala Spezifisch-Notwendigkeit und Adhärenz bei der Befragung nach 3 Monaten $(\mathrm{OR}=1.76$, $\mathrm{p}=0.156)$ und einem Jahr $(\mathrm{OR}=2.4, \mathrm{p}=0.065)$. Für die Patienten mit COPD zeigte sich eine ähnliche Tendenz der Assoziation zwischen Spezifisch-Notwendigkeit und Adhärenz, wobei die Assoziation bei der Befragung nach 3 Monaten signifikant war $(\mathrm{OR}=2.63, \mathrm{p}=0.005)$. Des Weiteren zeigte sich eine inverse Assoziation zwischen der Subskala Spezifisch-Befürchtungen und Medikamentenadhärenz. Für Patienten mit COPD war diese Assoziation bei der Erhebung nach drei Monaten $(\mathrm{OR}=0.55, \mathrm{p}=0.032)$ wie auch nach einem Jahr signifikant $(\mathrm{OR}=0.36$, $\mathrm{p}=0.011$ ). Die Untersuchung auf Multikollinearität ergab keine stark ausgeprägten Korrelationen zwischen den verschiedenen, in das multivariable Modell eingeschlossenen, Variablen. Die Sensitivitätsanalysen mit unterschiedlichen MARS Cut-Off Werten zur Definition adhärenten Verhaltens zeigten keine bedeutenden Unterschiede. Eine zusätzliche Sensitivitätsanalyse, welche nur die Teilnehmer der letzten Follow-Up Erhebung umfasste, zeigte darüber hinaus keine bedeutenden Abweichungen vom ursprünglichen Modell.

\section{Diskussion}

In der Baselineuntersuchung der Studie waren Allgemein-übermässiger Gebrauch und Allgemein-Schädlichkeit signifikant assoziiert mit einer niedrigen Chance, adhärent zu sein, während Spezifisch-Notwendigkeit signifikant mit Adhärenz assoziiert war. In der prospektiven Untersuchung zeigte sich die Assoziation zwischen den medikamentenbezogenen Überzeugungen und der Adhärenz als konsistent und mit der erwarteten Tendenz. Signifikant war die Assoziation jedoch nur bezüglich Spezifisch-Notwendigkeit und Spezifisch- 
Befürchtungen bei den Patienten mit COPD. Dies könnte darauf hinweisen, dass die Bedeutung des Einflusses von den medikamentenbezogenen Überzeugungen auf Adhärenz bei den beiden Krankheitsgruppen differiert. Der Vergleich der Ergebnisse von den beiden Krankheitsgruppen ist jedoch durch die Heterogenität der Studienpopulation limitiert. Für Patienten mit COPD war der Zusammenhang zwischen Spezifisch-Notwendigkeit und Adhärenz in der Follow-Up nach 3 Monaten positiv signifikant, dies konnte bereits in der Baselineuntersuchung gezeigt werden. Der Zusammenhang blieb in der Follow-Up nach einem Jahr positiv, erreichte aber nicht mehr das Signifikanzniveau. Ursächlich hierfür ist möglicherweise die geringe verbliebene Patientenzahl von nur 86 Patienten mit COPD. Patienten, die der Ansicht sind, dass sie ihre Medikamente benötigen, um ihr Leiden zu lindern, tendieren eher dazu, ihre Medikamente wie verordnet einzunehmen. Unsere Ergebnisse werden durch die Ergebnisse von George et al. unterstützt, welche in einer Studie mit 276 COPD Patienten zeigten, dass adhärente Patienten überzeugter waren von der Notwendigkeit ihrer Medikamente (37). In einer anderen Studie mit 188 Patienten mit COPD waren die Überzeugungen hinsichtlich Spezifisch-Notwendigkeit hingegen kein unabhängiger Einflussfaktor von Adhärenz (36). Die Spezifisch-Befürchtungen waren für die Gruppe mit COPD in beiden Follow-Up Untersuchungen signifikant invers mit Adhärenz assoziiert. Diese Ergebnisse stützen die Untersuchung von Krauskopf et al., die zeigten, dass höhere Scores auf der Spezifisch-Befürchtungen Subskala mit niedrigeren Chancen adhärenten Verhaltens assoziiert waren (36). In der Studie von Khdour et al. korrelierte adhärentes Verhalten mit den wahrgenommen Hindernissen in Bezug auf Medikation (insbesondere auch Nebenwirkungen). Dies unterstützt die These, dass SpezifischBefürchtungen die Chancen adhärenten Verhaltens beeinflussen (20). Eine andere Studie mit Patienten mit COPD zeigte, dass adhärente Patienten weniger Bedenken bezüglich ihrer Medikamente hatten als non-adhärente Patienten (37). Im Gegensatz zu unseren Ergebnissen zeigte eine vor kurzem veröffentlichte Studie mit Patienten mit COPD, die kürzlich aus dem Krankenhaus entlassen worden waren, keine Assoziation zwischen medikamentenbezogenen Überzeugungen und Adhärenz, welche mittels elektronischer Verfahren erfasst wurde (55).

Während in der Baselineerhebung verschiedene signifikante Assoziationen zwischen den medikamentenbezogenen Überzeugungen und dem Adhärenzverhalten bei den Patienten mit Asthma gezeigt werden konnten, beobachteten wir in der prospektiven Untersuchung ähnliche Tendenzen, jedoch ohne signifikante Zusammenhänge darstellen zu können. In der bereits erwähnten longitudinalen australischen Studie mit Patienten mit Asthma wurden medikamentenbezogene Überzeugungen nicht als Prädiktor von Medikamentenadhärenz 
identifiziert (38). Andere Studien zeigen jedoch eine positive Assoziation zwischen SpezifischNotwendigkeit und Adhärenz, wie es auch in unserer Studie gezeigt werden konnte $(34,35)$. Die Ergebnisse von Sofianou et al. stützen unsere Beobachtungen, dass Non-Adhärenz häufiger bei Patienten vorkam, welche weniger an die Notwendigkeit ihrer Medikamente glaubten und mehr Befürchtungen im Hinblick auf Nebenwirkungen hatten (21). Untersuchungen von Horne et al. zeigten positive Korrelationen zwischen Adhärenz und Spezifisch-Notwendigkeit und negative Korrelationen zwischen Adhärenz und Spezifisch-Befürchtungen bei Patienten mit Asthma $(25,26)$. Unsere Ergebnisse sind mit denen der eben genannten Studien vergleichbar. Das Signifikanzniveau wäre möglicherweise mit einer größeren Studienpopulation erreicht worden.

Das durchschnittliche Adhärenzniveau von 33\% in unserer Studie ähnelt den Ergebnissen einiger früherer Untersuchungen (19,37), andere Studien beschreiben jedoch ein höheres Adhärenzniveau (20,21,22,35). Diese Abweichungen sind möglicherweise durch unterschiedliche Messmethodik und unterschiedliche Studienpopulationen zu erklären. Das Adhärenzniveau der Patienten mit COPD lag in unserer Studie mit durchschnittlich 40\% deutlich höher als das bei den Patienten mit Asthma (ca. 17\%). Dieser Unterschied lässt sich möglicherweise durch die Zusammensetzung der Studienpopulation erklären: Unter den Patienten mit COPD war der Anteil an Patienten mit einer schweren COPD sehr hoch. Nur 6,3\% wurden dem GOLD Stadium I zugeordnet, je ein Drittel dem Stadium II und III und 28\% dem Stadium IV. Auch der mittlere COPD Assessment Test (CAT) Score von 23,26 (SD 7,2) zeigt, dass der Großteil der Patienten mit COPD sehr stark durch die Erkrankung beeinträchtigt wurde. Patienten mit einer sehr hohen Krankheitsbelastung sind möglicherweise auch adhärentere Patienten, da sie eventuell bemerken, dass die Medikamente ihnen Linderung verschaffen und Exazerbationen verhindern. Im Gegensatz dazu hatte ein Großteil der Patienten mit Asthma ein sehr gut kontrolliertes Asthma (mittlerer $A C Q$ Wert 1,5; SD 1,2). Es ist denkbar, dass die Einschränkungen dieser Patienten durch ihre Erkrankung deutlich geringer waren als die der Patienten mit COPD und daraus eine verminderte Adhärenz resultierte. Eine Studie zeigte zudem, dass Patienten mit Asthma generell dazu neigten, weniger adhärent zu sein als Patienten mit einer anderen chronischen Erkrankung (25). Die anfallsartige Symptomatik und die zwischenzeitlich z.T. komplette Beschwerdefreiheit der Asthmaerkrankung mögen dieses Verhalten bedingen.

Die Messung der Adhärenz stellt eine große Herausforderung dar. Es gibt zahlreiche Methoden, bisher hat sich jedoch keine als Goldstandard durchgesetzt (18). In unserer Studie wurde Adhärenz mittels Selbstbericht im Fragebogen gemessen. Diese Methodik wird oft als 
verzerrt kritisiert, da die soziale Erwünschtheit hierbei eine große Rolle spielen kann und daher Non-Adhärenz um bis zu 20\% unterschätzt wird (56). Es konnte jedoch andererseits gezeigt werden, dass selbst-berichtete Adhärenz häufig gut mit objektiv gemessener Adhärenz übereinstimmt (56). Des Weiteren sind die Fragen des MARS Fragebogens als negative Aussagen formuliert, um den Effekt erwünschten Antwortverhaltens zu reduzieren (57). Die soziale Erwünschtheit hat in unserer Studie womöglich vor allem bei der Baselineerhebung, welche in Kliniken und Praxen erfolgte, eine Rolle gespielt. Bei den Follow-Ups war ihr Einfluss möglicherweise geringer, da die Patienten die Fragebögen zuhause ausfüllten.

Wir erhoben keine Daten zur externen Validierung unserer Ergebnisse (z.B. mittels elektronischer Messverfahren), des Weiteren wurden keine Informationen bezüglich der Gründe gesammelt, warum Patienten die Studie abbrachen. Diese fehlenden Informationen limitieren die Aussagekraft unserer Studie.

Der Großteil unserer Patienten mit Asthma wurde ambulant rekrutiert, daher ist die Aussagekraft unserer Ergebnisse im Hinblick auf die Gesamtheit der Patienten mit Asthma eingeschränkt. In künftigen Untersuchungen sollten mehr Patienten mit schwerem Asthma eingeschlossen werden. Bei den Patienten mit COPD waren nur 6\% im Stadium GOLD 1, 70\% waren hospitalisierte Patienten. Aus diesem Grund sind unsere Ergebnisse nur eingeschränkt auf Patienten mit leichter COPD Erkrankung zu übertragen.

Unsere Studienergebnisse bestätigen die Bedeutung der medikamentenbezogenen Überzeugungen im Hinblick auf Medikamentenadhärenz bei Patienten mit COPD. Durch das prospektive Design kann eine vorsichtige Interpretation bezüglich der beobachteten Assoziation im Hinblick auf Kausalität erfolgen. Besonders die spezifischen medikamentenbezogenen Überzeugungen scheinen hierbei eine Rolle zu spielen, daher sollten sich Ärzte und Therapeuten primär auf diese fokussieren, um Adhärenz zu steigern. Durch das Erkennen der Ängste und der Überzeugungen der Patienten im Hinblick auf ihre Therapie, insbesondere ihre Medikamente, kann Non-Adhärenz möglicherweise reduziert werden. Randomisierte, kontrollierte Studien sind notwendig, um Interventionsmassnahmen zur Steigerung von Adhärenz zu prüfen. Zukünftige Untersuchungen sollten eine größere Patientengruppe sowie eine breitere Verteilung der Krankheitsschwere beinhalten. 


\section{Literaturverzeichnis}

1. Global Initiative for Asthma (GINA). Global Strategy for Asthma Management and Prevention Updated 2016. www.ginasthma.org (zuletzt besucht Dezember 2016).

2. Global Initiativ for Chronic Obstructive Lung Disease. Global strategy for the diagnosis, management and prevention of COPD (updated 2016) 2016.

3. Gibson GJ, Loddenkemper R, Sibille Y, et al. The European Lung White Book: Respiratory Health and Disease in Europe; 2013.

4. Barnes PJ. The size of the problem of managing asthma. Respiratory Medicine 2004; 98:S4-S8.

5. Masoli M, Fabian D, Holt S, et al. The global burden of asthma: executive summary of the GINA Dissemination Committee report. Allergy 2004; 59(5):469-78.

6. Mathers CD, Loncar D. Projections of Global Mortality and Burden of Disease from 2002 to 2030. PLoS Medicine 2006; 3:e442.

7. Halbert RJ, Natoli JL, Gano A, et al. Global burden of COPD: systematic review and metaanalysis. Eur Respir J 2006; 28(3):523-32.

8. Buist AS, McBurnie MA, Vollmer WM, et al. International variation in the prevalence of COPD (The BOLD Study): A population-based prevalence study. The Lancet 2007; 370(9589):741-50.

9. Geldmacher H, Biller H, Herbst A, et al. Die Pravalenz der chronisch obstruktiven Lungenerkrankung (COPD) in Deutschland. Ergebnisse der BOLD-Studie. Dtsch Med Wochenschr 2008; 133(50):2609-14.

10. Raadts, A., Juergens, U. Volkswirtschaftliche Bedeutung von Asthma und COPD. In: Lingner H, Schwartz F-W, Schultz K, editors. Volkskrankheit Asthma/ COPD. Heidelberg: Springer; 2007. p. 37-48.

11. Accordini S, Bugiani M, Arossa W, et al. Poor control increases the economic cost of asthma. A multicentre population-based study. Int Arch Allergy Immunol 2006; 141(2):18998 .

12. Sullivan SD, Rasouliyan L, Russo PA, et al. Extent, patterns, and burden of uncontrolled disease in severe or difficult-to-treat asthma. Allergy 2007; 62(2):126-33.

13. Wacker ME, Jorres RA, Schulz H, et al. Direct and indirect costs of COPD and its comorbidities: Results from the German COSYCONET study. Respiratory Medicine 2016; 111:39-46.

14. Bateman ED, Boushey HA, Bousquet J, et al. Can guideline-defined asthma control be achieved? The Gaining Optimal Asthma ControL study. Am J Respir Crit Care Med 2004; 170(8):836-44.

15. Calverley P, Pauwels R, Vestbo J, et al. Combined salmeterol and fluticasone in the treatment of chronic obstructive pulmonary disease: A randomised controlled trial. The Lancet 2003; 361(9356):449-56.

16. Suissa S, Ernst P, Kezouh A. Regular use of inhaled corticosteroids and the long term prevention of hospitalisation for asthma. Thorax 2002; 57(10):880-4. 
17. Bourbeau J, Bartlett SJ. Patient adherence in COPD. Thorax 2008; 63(9):831-8.

18. Sumino K, Cabana MD. Medication adherence in asthma patients. Curr Opin Pulm Med 2013; 19(1):49-53.

19. Mehuys E, Boussery K, Adriaens E, et al. COPD management in primary care: an observational, community pharmacy-based study. Ann Pharmacother 2010; 44(2):257-66.

20. Khdour MR, Hawwa AF, Kidney JC, et al. Potential risk factors for medication nonadherence in patients with chronic obstructive pulmonary disease (COPD). Eur J Clin Pharmacol 2012; 68(10):1365-73.

21. Sofianou A, Martynenko M, Wolf MS, et al. Asthma beliefs are associated with medication adherence in older asthmatics. J Gen Intern Med 2013; 28(1):67-73.

22. Menckeberg TT, Bouvy ML, Bracke M, et al. Beliefs about medicines predict refill adherence to inhaled corticosteroids. J Psychosom Res 2008; 64(1):47-54.

23. Adherence to long-term therapies: evidence for action. World Health Organization 2003. www.who.int/chp/knowledge/publications/adherence_full_report.pdf (zuletzt besucht April 2017).

24. Gillissen A, Busch K, Juergens U. Adherence to therapy in bronchial asthma. Dtsch Med Wochenschr 2007; 132(23):1281-6.

25. Horne R, Weinman J. Patients' beliefs about prescribed medicines and their role in adherence to treatment in chronic physical illness. Journal of Psychosomatic Research 1999; 47(6):555-67.

26. Horne R, Weinman J. Self-regulation and Self-management in Asthma: Exploring The Role of Illness Perceptions and Treatment Beliefs in Explaining Non-adherence to Preventer Medication. Psychology \& Health 2002; 17(1):17-32.

27. Horne R. Representations of Medication and Treatment: Advances in Theory and Measurement. In: Petrie KJ, Weinman JA, editors. Perceptions of Health and Illness: Current Research and Applications. Amsterdam: Harwood; 1997. p. 155-88.

28. Nestoriuc Y, Glombiewski JA, Horne R, et al. Beeinflusst die Einstellung zu Medikamenten deren Einnahme? Validierung des "Beliefs about Medicines" Fragebogens in der Allgemeinbevölkerung. Zeitschrift für Klinische Psychologie und Psychotherapie 2010; 39:64-9.

29. Horne R, Weinman J, Hankins M. The beliefs about medicines questionnaire: The development and evaluation of a new method for assessing the cognitive representation of medication. Psychology \& Health 1999; 14(1):1-24.

30. Diefenbach MA, Leventhal H. The common-sense model of illness representation: Theoretical and practical considerations. Journal of Social Distress and the Homeless 1996; $5(1): 11-38$.

31. Horne R, Chapman SCE, Parham R, et al. Understanding Patients' Adherence-Related Beliefs about Medicines Prescribed for Long-Term Conditions: A Meta-Analytic Review of the Necessity-Concerns Framework. PLoS One 2013; 8(12):e80633.

32. Mahler C, Hermann K, Horne R, et al. Patients' beliefs about medicines in a primary care setting in Germany. J Eval Clin Pract 2012; 18(2):409-13. 
33. Phatak HM, Thomas J. Relationships between beliefs about medications and nonadherence to prescribed chronic medications. Ann Pharmacother 2006; 40(10):1737-42.

34. Emilsson M, Berndtsson I, Lotvall J, et al. The influence of personality traits and beliefs about medicines on adherence to asthma treatment. Prim Care Respir J 2011; 20(2):141-7.

35. van Steenis M, Driesenaar J, Bensing J, et al. Relationship between medication beliefs, self-reported and refill adherence, and symptoms in patients with asthma using inhaled corticosteroids. Patient Prefer Adherence 2014; 8:83-91.

36. Krauskopf K, Federman AD, Kale MS, et al. Chronic Obstructive Pulmonary Disease Illness and Medication Beliefs are Associated with Medication Adherence. COPD 2015; 12(2):151-64.

37. George J, Kong DCM, Thoman R, et al. Factors associated with medication nonadherence in Patients with COPD. Chest 2005; 128(5):3198-204.

38. Ovchinikova L, Smith L, Bosnic-Anticevich S. Inhaler technique maintenance: gaining an understanding from the patient's perspective. J Asthma 2011; 48(6):616-24.

39. Mahler C, Hermann K, Horne R, et al. Assessing reported adherence to pharmacological treatment recommendations. Translation and evaluation of the Medication Adherence Report Scale (MARS) in Germany. J Eval Clin Pract 2010; 16(3):574-9.

40. Opitz U, Glattacker M, Bengel J, et al. Der "Beliefs about Medicines Questionnaire" (BMQ ( ) R Horne) - Übersetzung und erste methodische Prüfung an Patienten mit Fibromyalgie. In: Deutsche Rentenversicherung Bund, eds. Internetausgabe des Tagungsbandes zum 17. Rehabilitationswissenschaftlichen Kolloquium 2008. www.eservicedrv.de/ForschPortalWeb/ressource?key=tagungsband_17_reha_kolloqu.pdf\#page=100 (zuletzt besucht April 2017).

41. Jones PW, Harding G, Berry P, et al. Development and first validation of the COPD Assessment Test. European Respiratory Journal 2009; 34(3):648-54.

42. Juniper EF, O'byrne PM, Guyatt G, et al. Development and validation of a questionnaire to measure asthma control. Eur Respir J 1999; 14(4):902.

43. COPD Assessment Test. Healthcare Professional User Guide: Expert Guidance on frequently Asked Questions.

www.catestonline.org/images/UserGuides/CAT_HCP\%20User\%20Guide.pdf (zuletzt besucht April 2017).

44. Ferrer M. Chronic Obstructive Pulmonary Disease Stage and Health-Related Quality of Life. Ann Intern Med 1997; 127(12):1072.

45. Chen H, Gould MK, Blanc PD, et al. Asthma control, severity, and quality of life: quantifying the effect of uncontrolled disease. J Allergy Clin Immunol 2007; 120(2):396-402.

46. Gandek B, Ware JE, Aaronson NK, et al. Cross-Validation of Item Selection and Scoring for the SF-12 Health Survey in Nine Countries: Results from the IQOLA Project. J Clin Epidemiol 1998; 51(11):1171-8.

47. Luszczynska A, Scholz U, Schwarzer R. The general self-efficacy scale: multicultural validation studies. J Psychol 2005; 139(5):439-57.

48. Goodwin RD, Fergusson DM, Horwood LJ. Asthma and depressive and anxiety disorders among young persons in the community. Psychol. Med. 2004; 34(08):1465. 
49. Krauskopf KA, Sofianou A, Goel MS, et al. Depressive symptoms, low adherence, and poor asthma outcomes in the elderly. J Asthma 2013; 50(3):260-6.

50. Yohannes AM, Willgoss TG, Baldwin RC, et al. Depression and anxiety in chronic heart failure and chronic obstructive pulmonary disease: prevalence, relevance, clinical implications and management principles. Int J Geriatr Psychiatry 2010; 25(12):1209-21.

51. DiMatteo MR, Lepper HS, Croghan TW. Depression Is a Risk Factor for Noncompliance With Medical Treatment. Arch Intern Med 2000; 160(14):2101.

52. Bosley CM, Fosbury JA, Cochrane GM. The psychological factors associated with poor compliance with treatment in asthma. Eur Respir J 1995; 8:899-904.

53. Zigmond AS, Snaith RP. The Hospital Anxiety and Depression Scale. Acta Psychiatr Scand 1983; 67(6):361-70.

54. Brandstetter S, Finger T, Fischer W, et al. Differences in medication adherence are associated with beliefs about medicines in asthma and COPD. Clin Transl Allergy 2017; 7:39.

55. Sulaiman I, Cushen B, Greene G, et al. Objective Assessment of Adherence to Inhalers by Patients with Chronic Obstructive Pulmonary Disease. Am J Respir Crit Care Med 2017; 195(10):1333-43.

56. Haynes RB, Taylor D, Sackett D, et al. Can Simple Clinical Measurements Detect Patient Noncompliance? Hypertension 1980; 2:757-64.

57. Cohen JL, Mann DM, Wisnivesky JP, et al. Assessing the validity of self-reported medication adherence among inner-city asthmatic adults: the Medication Adherence Report Scale for Asthma. Ann Allergy Asthma Immunol 2009; 4(103):325-31. 


\section{Eidesstattliche Erklärung}

Ich erkläre,

- dass ich den Doktorgrad der Medizin nicht schon an einer Hochschule der Bundesrepublik Deutschland erworben habe

- dass ich nicht an anderer Stelle zu einem Promotionsverfahren zum Erwerb des medizinischen Doktorgrades zugelassen bin

- dass ich die medizinische Doktorprüfung nicht schon an einer Hochschule der Bundesrepublik Deutschland endgültig nicht bestanden habe

Außerdem erkläre ich,

- dass mir keine Tatsachen bekannt sind, die mich zur Führung eines akademischen Grades im Sinne des Gesetzes über die Führung akademischer Grade unwürdig erscheinen lassen

- dass ich die vorliegende Arbeit ohne unzulässige Hilfe Dritter und ohne Benutzung anderer als der angegebenen Hilfsmittel angefertigt habe. Die aus anderen Quellen direkt oder indirekt übernommenen Daten und Konzepte sind unter Angabe der Quelle gekennzeichnet. Insbesondere habe ich nicht die entgeltliche Hilfe von Vermittlungs- bzw. Beratungsdiensten (Promotionsberater oder andere Personen) in Anspruch genommen. Niemand hat von mir unmittelbar oder mittelbar geldwerte Leistungen für Arbeit erhalten, die im Zusammenhang mit dem Inhalt der vorgelegten Dissertation stehen. Die Arbeit wurde bisher weder im Innoch im Ausland in gleicher oder ähnlicher Form einer anderen Prüfungsbehörde vorgelegt.

Roggwil, 10.04.2018

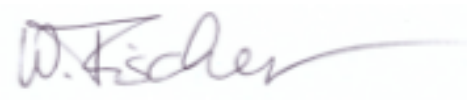

( Unterschrift ) 


\section{Danksagung}

Ich möchte mich bei allen denjenigen Personen bedanken, die an der Entstehung dieser Doktorarbeit mitgewirkt haben, insbesondere gilt mein Dank dabei Herrn Prof. Dr. Christian Apfelbacher, der mich auf dem langen Weg stets tatkräftig und kompetent unterstützt hat und durch dessen Zuspruch auch schwierige Phasen der Studie überwunden werden konnten.

Bedanken möchte ich mich auch bei Frau Dr. Susanne Brandstetter für Ihre ständigen Bemühungen und ihre tatkräftige und unermüdliche Unterstützung während der gesamten Dauer dieser Doktorarbeit, durch welche ich mich stets sehr gut betreut gefühlt habe.

Ich bedanke mich bei Herrn Prof. Dr. Michael Pfeifer für sein Engagement für diese Studie und für die Möglichkeit, dass in seiner Abteilung für Pneumologie ein Großteil der Patienten rekrutiert werden konnte.

Mein Dank gilt auch den Mitarbeitern der Abteilung Medizinische Soziologie des Lehrstuhls Epidemiologie und Präventivmedizin der Universität Regensburg, welche an dieser Doktorarbeit mitgewirkt haben.

Bedanken möchte ich mich auch bei Tamara Finger für ihren tatkräftigen Beitrag zur Erhebung und Eingabe der Studiendaten sowie für ihre Unterstützung besonders in der Anfangsphase der Doktorarbeit.

Abschließend gilt ein besonderer Dank meinem privaten Umfeld, insbesondere meiner Familie für die Unterstützung und den unermüdlichen Zuspruch, welche mir besonders in schwierigen Phasen Mut und Kraft verliehen haben. 




\title{
Specific, but not general beliefs about medicines are associated with medication adherence in patients with COPD, but not asthma: Cohort study in a population of people with chronic pulmonary disease ${ }^{i^{2}}$
}

\author{
Wiebke Fischer ${ }^{\mathrm{a}}$, Susanne Brandstetter ${ }^{\mathrm{a}}$, Magdalena Brandl ${ }^{\mathrm{a}}$, Tamara Finger ${ }^{\mathrm{a}}$, \\ Merle M. Böhmer ${ }^{\mathrm{a}, \mathrm{b}}$, Michael Pfeifer ${ }^{\mathrm{c}}$, Christian Apfelbacher ${ }^{\mathrm{a}, *}$ \\ a Medical Sociology, Institute of Epidemiology and Preventive Medicine, University of Regensburg, Germany \\ ${ }^{\mathrm{b}}$ Department of Public Health Microbiology \& Infectious Disease Epidemiology, Bavarian, Health and Food Safety Authority, Oberschleissheim, Germany \\ ${ }^{\mathrm{c}}$ Department of Pneumology, Klinik Donaustauf, Donaustauf, Germany
}

\section{A R T I C L E I N F O}

\section{Keywords:}

Asthma

Beliefs about medicines

Chronic obstructive pulmonary disease COPD

Medication adherence

\begin{abstract}
A B S T R A C T
Background: Beliefs about medicines are regarded as influencing factors on medication adherence (Horne, 1997). Adherence levels in patients with chronic pulmonary diseases are low (Bourbeau and Bartlett, 2008; Sumino and Cabana, 2013). A better understanding of the predictive role of patients' beliefs about medicines for adherence might be a crucial step to improve medication adherence.

Objective: This prospective study investigated the association between beliefs about medicines and medication adherence in patients with asthma and COPD.

Methods: The Beliefs about Medicines Questionnaire (BMQ) and the Medication Adherence Rating Scale (MARS) were administered to 402 patients (49\% asthma, 51\% COPD, 50\% female, mean age 56.7 years (SD = 15.9)) at baseline. Follow-ups were carried out after $3(\mathrm{~N}=255)$ and 12 months $(\mathrm{N}=171)$. Multivariable logistic regression analyses were performed analysing the association between the BMQ subscales at baseline and adherence at each follow-up inquiry. Sociodemographic, psychosocial, and disease related factors were considered as potential confounders.

Results: One third of the patients showed adherent behavior ( $18 \%$ and $46 \%$ of people with asthma and COPD). In the COPD sample, the subscale Specific-necessity showed a significant positive association with adherence at the 3-months-follow-up (OR $=2.6,95 \%$ CI 1.4-5.1) and the subscale Specific-concerns showed a significant inverse association with adherence at the 3-months-follow-up ( $\mathrm{OR}=0.6,95 \% \mathrm{CI} 0.3-0.95$ ) and the 12-months-followup (OR $=0.4,95 \%$ CI 0.2-0.8). No significant association was found for the asthmatic sample.

Conclusions: Beliefs about medicines are important factors predicting future medication adherence in patients with COPD, but not asthma. Physicians should primarily focus on the specific beliefs of their patients in order to diminish medication non-adherence.
\end{abstract}

\section{Introduction}

Asthma and COPD are chronic pulmonary diseases with a significant airflow obstruction being reversible in asthma [4] and constant in COPD [5]. Cough and shortness of breath are major symptoms affecting both types of patients either with COPD [6] or asthma [5]. As chronic diseases, asthma and COPD present a substantial economic burden on our health system [7].

Treatment regimes in both diseases involve pharmacological (corticosteroids and bronchodilatators), and non-pharmacological strategies (e.g. smoking cessation) [4,5]. Improvement of health status can be obtained by optimal therapy management in both groups of patients [8], reducing risks of exacerbations [9], and the number of hospital admissions [10]. Effective therapy management requires patients with asthma or COPD to adhere to the medication prescribed. However, adherence has been found to be low in patients with chronic pulmonary diseases such as asthma and COPD [2,3], ranging from 40 to $70 \%$ in different studies [11-14]. In order to identify ways to improve

\footnotetext{
Work was conducted at the Medical Sociology unit of the Institute of Epidemiology and Preventive Medicine at the University of Regensburg, Germany.

* Corresponding author at: Medical Sociology, Department for Epidemiology and Preventive Medicine, University of Regensburg, Dr.-Gessler-Str. 17, 93051 Regensburg, Germany.

E-mail addresses: fischerwiebke@gmx.de (W. Fischer), Susanne.Brandstetter@klinik.uni-regensburg.de (S. Brandstetter), Magdalena.Brandl@klinik.uni-regensburg.de (M. Brandl), tamara.finger@gmx.de (T. Finger), merle.boehmer@yahoo.de (M.M. Böhmer), Michael.Pfeifer@klinik.uni-regensburg.de (M. Pfeifer),

Christian.Apfelbacher@klinik.uni-regensburg.de (C. Apfelbacher).
} 
adherence in patients with chronic pulmonary disease, it is very important to investigate factors influencing adherence.

Sociodemographic factors (e.g. sex, age, education), clinical (duration of illness) and care-related variables (number of hospital visits) only correlate weakly with reported adherence [12,15,16].

Patients' illness perception and beliefs that people with chronic disease hold about their medicines have moved into the focus of current adherence to treatment research [1]. The concept of these beliefs is derived from different conceptual models. One of them is the common sense model that states that a patient has to deal with two different aspects regarding the illness representation. On the one hand he or she has to deal with the perceived health threat of the disease (cognitive level) and on the other hand with the emotional reactions to this threat (emotional level). The processing of these aspects is further influenced by psychological and biological aspects of the individual and by sociocultural aspects among them beliefs about medication [17]. Another concept of adherence-related beliefs is the Necessity-concerns framework which suggests that patients' adherence depends mainly on two aspects: their perception of their personal need for treatment (necessity beliefs) and their fear of potential negative consequences [18]. According to this framework Horne structured beliefs about medicines into five domains: Specific-necessity, Specific-concerns, General-harm, General-overuse and General-utility [1,19], represented in the Beliefs about Medicines Questionnaire [20]. These beliefs have been used to predict medication adherence in chronically ill patients [15,21].

In asthma, patients' concerns about their medication as well as their perception that medication is generally harmful and overused have been linked to lower levels of adherence [15,16,22]. Further studies showed a positive relationship between Specific-necessity and medication adherence in patients with asthma [14,23,24], and a higher risk of non-adherence in patients who were more concerned about their asthma medication [13]. For COPD patients, fewer studies have investigated the association of beliefs about medicines and medication adherence $[12,25,26]$. Supporting the findings of research in patients with asthma, their results showed that higher concerns increase the risk of non-adherence while patients with a stronger belief in the necessity of their medication reported being adherent more often.

A better understanding of the role beliefs about medicines play for medication adherence of patients with asthma and COPD could be a crucial step in the improvement of patients' medication adherence and consequently, health and cost outcomes [27].

We could find only one other prospective study investigating factors influencing adherence in respiratory disease and the focus of this study was on inhaler maintenance in patients with asthma in Australia [28]. We did not identify any prospective study with a follow-up period beyond one month nor in patients with COPD. However, more longitudinal studies are warranted to analyse a potentially causal relationship of the association between medication beliefs and adherence and also because behavior change is a dynamic process [14].

Therefore, the aim of this study was to investigate the association between beliefs about medicines and medication adherence in patients with COPD and asthma in a prospective manner. In particular, we wanted to test the hypotheses that the scores of the BMQ subscales Specific-concerns, General-overuse and General-harm are inversely associated and the scores of the subscales General-utility and Specificnecessity are positively associated with medication adherence.

\section{Material and methods}

\subsection{Study design}

For the baseline survey, patients with COPD and asthma were recruited from June 2013 to December 2014 from different hospitals, primary care and specialists practices in the area of Regensburg, Germany. Inclusion criteria were: main diagnosis of asthma or COPD made by a physician; disease duration at least 3 months; age $\geq 18$ years. Patients without sufficient understanding of the German language and with acute psychiatric and neurological diseases (except for depression and anxiety disorders) were excluded. Patients' written informed consent was obtained. Data was collected by means of questionnaires and from patients' medical files. Follow-ups were performed after three (September 2013 to March 2015) and 12 months (June 2014 to December 2015). Questionnaires were sent by post. Reminder were sent by post. Patients were contacted by telephone if no response was obtained after sending the reminder in order to gain a good sample for both follow-up inquiries.

\subsection{Data collection}

\subsubsection{Outcome measure: adherence to medication}

At each point in time of our longitudinal study (baseline, after 3 months, after 12 months) adherence to medication was measured by the German version of the Medication Adherence Report Scale (MARS) which has been described as an appropriate questionnaire to detect non-adherence [29]. Each of the five items of the MARS is scored from 1 (always) to 5 (never). The adherence score is derived from the sum of the five item scores. As the MARS scores were skewed to the right, we dichotomized adherence. A score of 25 defines adherence, a score < 25 non-adherence according to George et al. [26]. Sensitivity analyses with cut-off MARS scores of 23 and 22 were performed.

\subsubsection{Explanatory variables: beliefs about medicines}

The German version of the Beliefs about Medicines Questionnaire (BMQ) [20,30] was administered, which has been shown as an appropriate instrument to measure patients' beliefs about medicines [21]. The BMQ is a 23-item questionnaire with five scales: The BMQ Specificnecessity (patients' beliefs about how important their personal medication is for their health), the BMQ Specific-concerns (patients' worries about negative effects of their medication), the BMQ General-overuse (addressing the statement that medicine is overused), the BMQ Generalharm (addressing beliefs that medication is harmful and poisonous) and the BMQ General-utility (addressing beliefs that medication is useful to improve patients' health status) $[19,20]$. Each item is scored on a 5point Likert scale. A lower score indicates disagreement, while a higher score indicates agreement [20]. BMQ subscale scores were calculated as mean scores of corresponding items and range from 1 to 5 . The mean scores of subscale scores were used for statistical analysis.

\subsubsection{Potentially confounding variables}

2.2.3.1. Patients' characteristics. Sociodemographic data (age, sex, level of education, living situation) was collected. Educational level was subdivided into the categories low ( $<10$ years of school attendance), medium ( 10 years of school attendance) and high ( $>10$ years of school attendance). At baseline, the number of current smokers and the number of daily cigarettes was collected for patients with COPD only, but was asked for both groups at follow-up one and two.

2.2.3.2. Disease characteristics. Information about the time since the diagnosis of asthma or COPD as well as the number of comorbidities were collected. At baseline, information on the number of exacerbations in the last months was collected for patients with COPD only and for both groups of patients at the follow-ups. The presence of side effects was collected at each inquiry for all patients.

Asthma and COPD severity was measured using the forced expiratory volume in $1 \mathrm{~s}\left(\mathrm{FEV}_{1}\right)$, the COPD assessment test (CAT) [31] and the Asthma Control Questionnaire (ACQ) [32]. COPD severity was classified into the four GOLD categories according to the Global Initiative for Chronic Obstructive Lung Disease (GOLD) [5].

The CAT consists of eight items with six categories $(0=$ no impairment, $5=$ strong impairment) that cover a broad range of COPD associated symptoms and effects on patients' lives [31]. Studies describe it as a reliable measure of COPD severity [31] with good validity [33]. 
The CAT score is derived from the sum of the eight items and ranges from 0 to 40 . A CAT score $<10$ indicates a low impact level, a score from 10 to 20 indicates a medium level and a CAT score $>20$ indicates a high impact level of the disease [34]. For statistical analysis mean scores of CAT scores were used.

The ACQ consists of seven items (six questions on a 7-point scale [0 = no impairment; $6=$ maximum impairment $]$ and the predicted $\mathrm{FEV}_{1}$ ) addressing symptoms, impairment and use of reliever medication [32]. Each item is equally weighted and the ACQ score is derived from the mean of the seven items, ranging from 0 (totally controlled) to 6 (extremely poorly controlled) [35]. Studies revealed that the ACQ has good measurement properties and high reliability [32]. The mean ACQ score was used for statistical analysis.

2.2.3.3. Treatment-related characteristics. The distance to the physician in minutes, the number of doctor's visits in the last months, the number of medication (inhaler, pills and others) and information about the treatment setting were collected.

\subsubsection{Psychosocial constructs.}

\section{- Quality of life}

The Short-Form Health Survey Questionnaire 12 (SF-12) consists of 12 items in two scales: physical component scale (PCS) and mental component scale (MCS) [36]. Response categories differ for each item (binary or 3- to 5-point Likert scales). We calculated both scores according to Ware et al. [37]. Low scores with a minimum of 0 indicate low quality of life while higher scores (maximum of 100) indicate higher quality of life. A mean score of 50 is found in the general population [37]. Mean scores of both scales were used for statistical analysis.

\section{- Self-efficacy}

Perceived self-efficacy was measured by the General Self-Efficacy Scale (GSE). The scale includes 10 items, each of them scoring from 1 (indicating that the item's statement is incorrect) to 4 (indicating that the item's statement is absolutely correct). Total score ranges from 10 to 40. A higher score indicates higher self-efficacy. Studies confirmed the validity of the GSE as a psychometric scale to measure self-efficacy [38].

\section{- Depression and anxiety}

Patients completed the German version of the Hospital Anxiety and Depression scale (HADS) [39]. The HADS consists of a depression and an anxiety subscale with 7 items each and has been shown to be an appropriate measure to assess symptom severity and caseness of anxiety disorders and depression [40]. Scores of the subscales are derived from the sum of the seven items and range from 0 to 21 . There are three possible categories: A score from 0 to 7 indicates a low probability of clinically significant anxiety or depression (inconspicuous). A score from 8 to 10 indicates doubtful cases (marginally conspicuous) and a score of 11 to 21 indicates a high probability of significant anxiety or depression (conspicuous) [39].

\subsection{Statistical analysis}

Statistical analysis was carried out separately for asthma and COPD patients using IBM SPSS Statistics 24.0. Patients with missing data were excluded only from the analysis the data was relevant for but were not excluded listwise from the other analyses. The level of significance was set at 0.05. Mann-Whitney and Fisher's exact tests were used to compare characteristics of patients who did and did not participate in the one-year-follow-up in order to identify potential risk factors of loss to follow-up. The statistical analysis of this paper focused on analysing the prospective associations. Results of the analysis of the associations at baseline have been published elsewhere [41]. For each follow-up and illness group, multivariable logistic regression analysis was performed for each baseline BMQ subscale score as explanatory and adherence as dichotomous, dependent variable. Univariate analyses were performed in order to identify variables which were potentially confounding variables of the association between beliefs about medicines at baseline and medication adherence at each point in time. Potentially confounding variables were patient, disease and treatment characteristics as well as psychosocial constructs as assessed at baseline. If there was an association $(\mathrm{p} \leq .20)$ of the baseline variable with adherence the relevant variable was entered in the multivariable analysis for this point in time. All variables were tested for multicollinearity using the variance inflation factor (VIF) and the tolerance statistic. In order to make the models comparable, baseline variables that were not collected for both illness groups were excluded from the multivariable logistic regression analysis despite their univariate association with the outcome variable. A sensitivity analysis of the final models was performed by including the patients that participated in the last follow-up only.

\section{Results}

\subsection{Study population}

A total number of 402 patients participated in the study of which almost half had asthma. Almost half were female, mean age was 56.7 years (SD 15.9). The majority of participants (84.2\%) had a low or medium level of education. $>60 \%$ of participants were outpatients (95.4\% and $30.6 \%$ of patients with asthma and COPD). About two thirds of the patients with COPD were classified as stage GOLD II (32.2\%) and stage GOLD III (33.6\%). 28\% were classified as GOLD IV, whereas only $6.3 \%$ of all COPD patients belonged to stage GOLD I. The mean CAT score was 23.3 (SD 7.2) indicating a high impact level of the disease in most COPD patients. A good level of disease control in most asthma patients was indicated by a mean ACQ score of 1.5 (SD 1.2). For further characteristics of study population see Table 1 .

255 patients $(49.4 \%$ female) participated in the three months follow-up (43.5\% of them having asthma). 171 patients $(49.7 \%$ female) remained in the study after one year $(42.7 \%$ with asthma). Characteristics of patients who participated in the one year follow-up showed only two significant differences from those patients who did not participate: patients who participated in the follow-up were older $(\mathrm{Mdn}=62.3, Q 1 / Q 3=53.8 / 67.7)$ than those who did not participate $(M d n=57.7, Q 1 / Q 3=41.5 / 67.6, U=22.453, p=0.019)$ and visited the physician more frequently in the previous 12 months $(M d n=4, Q 1 / Q 3=2 / 6)$ than those patients who did not participate $(M d n=3, Q 1 / Q 3=2 / 5, U=21.151, p=.007)$. There was no significant difference between the baseline adherence behavior of patients who did and did not participate in the last follow-up.

\subsection{Medication adherence}

At three months follow-up, a total of $30.6 \%$ of the patients reported to be adherent. $>40 \%$ of the patients with COPD reported adherent behavior (43\%). In patients with asthma only $14.5 \%$ were found to be adherent at this point. About one third (33.9\%) of participants indicated being adherent at the follow-up after one year. In patients with asthma $17.8 \%$ were adherent, in patients with COPD $45.9 \%$ were adherent. Table 2 shows the MARS scores at each point in time.

\subsection{Beliefs about medicines}

BMQ subscales scores at baseline were particularly high for General-utility $(\mathrm{M}=3.9, \mathrm{SD}=0.68)$ and Specific-necessity $(\mathrm{M}=3.9$, $\mathrm{SD}=0.90)$, and were low for Specific-concerns $(\mathrm{M}=2.4, \mathrm{SD}=.78)$ 
Table 1

Main characteristics of study population at baseline survey.

\begin{tabular}{|c|c|c|c|c|}
\hline & & Asthma & COPD & Total sample \\
\hline Total number of participants & N (\%) & $196(48.8)$ & $206(51.2)$ & $402(100.0)$ \\
\hline Age & $\mathrm{M} \pm \mathrm{SD}$ & $47.70 \pm 16.69$ & $65.30 \pm 8.85$ & $56.72 \pm 15.91$ \\
\hline Gender & $\mathrm{N}(\%)$ & & & \\
\hline Males & & $78(39.8)$ & $125(60.7)$ & $203(50.5)$ \\
\hline Females & & $118(60.2)$ & $81(39.3)$ & 199 (49.5) \\
\hline Level of education & $\mathrm{N}(\%)$ & & & \\
\hline Low & & $77(40.3)$ & $145(74.4)$ & $222(57.5)$ \\
\hline Medium & & $63(33.0)$ & $40(20.5)$ & $103(26.7)$ \\
\hline High & & $51(26.7)$ & $10(5.1)$ & $61(15.8)$ \\
\hline Number of adults living in the same household & $\mathrm{M} \pm \mathrm{SD}$ & $1.74 \pm 1.31$ & $1.35 \pm 1.06$ & $1.54 \pm 1.20$ \\
\hline Treatment & $\mathrm{N}(\%)$ & & & \\
\hline Outpatient & & $187(95.4)$ & $63(30.6)$ & $250(62.2)$ \\
\hline Inpatient & & $9(4.6)$ & $143(69.4)$ & $152(37.8)$ \\
\hline Duration of disease (years) & $\mathrm{M} \pm \mathrm{SD}$ & $11.49 \pm 9.89$ & $8.44 \pm 7.92$ & $9.94 \pm 9.06$ \\
\hline Distance to the physician (minutes) & $\mathrm{M} \pm \mathrm{SD}$ & $24.17 \pm 16.74$ & $30.11 \pm 18.05$ & $27.18 \pm 17.65$ \\
\hline Medication & $\mathrm{M} \pm \mathrm{SD}$ & & & \\
\hline Number of pills & & $0.83 \pm 1.95$ & $4.48 \pm 3.65$ & $2.71 \pm 3.47$ \\
\hline Number of inhaler medications & & $1.72 \pm 0.88$ & $2.06 \pm 0.99$ & $1.90 \pm 0.95$ \\
\hline Number of other medications & & $0.10 \pm 0.43$ & $0.36 \pm 0.71$ & $0.24 \pm 0.61$ \\
\hline Side effects & N (\%) & $16(8.3)$ & $48(24.2)$ & $64(16.4)$ \\
\hline $\mathrm{FEV}_{1} \%$ & $\mathrm{M} \pm \mathrm{SD}$ & $89.19 \pm 22.06$ & $46.89 \pm 21.44$ & $70.35 \pm 30.27$ \\
\hline GOLD Classification & N (\%) & & & \\
\hline GOLD I & & & $9(6.3)$ & \\
\hline GOLD II & & & $46(32.2)$ & \\
\hline GOLD III & & & $48(33.6)$ & \\
\hline GOLD IV & & & $40(28.0)$ & \\
\hline COPD Assessment Test (CAT) & $\mathrm{M} \pm \mathrm{SD}$ & & $23.26 \pm 7.20$ & \\
\hline Asthma Control Questionnaire (ACQ) & $\mathrm{M} \pm \mathrm{SD}$ & $1.50 \pm 1.22$ & & \\
\hline \multicolumn{5}{|l|}{ BMQ } \\
\hline General-overuse & & $3.14 \pm 0.74$ & $3.17 \pm 0.74$ & $3.15 \pm 0.74$ \\
\hline General-harm & $\mathrm{M} \pm \mathrm{SD}$ & $2.38 \pm 0.64$ & $2.48 \pm 0.70$ & $2.43 \pm 0.67$ \\
\hline General-utility & & $3.92 \pm 0.64$ & $3.89 \pm 0.72$ & $3.90 \pm 0.68$ \\
\hline Specific-necessity & & $3.53 \pm 0.96$ & $4.15 \pm 0.73$ & $3.85 \pm 0.90$ \\
\hline Specific-concerns & & $2.20 \pm 0.76$ & $2.56 \pm 0.76$ & $2.38 \pm 0.78$ \\
\hline SF-12 & $\mathrm{M} \pm \mathrm{SD}$ & & & \\
\hline Physical component summary & & $46.46 \pm 10.78$ & $31.78 \pm 9.71$ & $39.02 \pm 12.61$ \\
\hline Mental component summary & & $43.97 \pm 12.44$ & $37.99 \pm 14.33$ & $40.94 \pm 13.74$ \\
\hline HADS & $\mathrm{M} \pm \mathrm{SD}$ & & & \\
\hline Depression subscale & & $5.23 \pm 4.15$ & $8.28 \pm 4.66$ & $6.76 \pm 4.66$ \\
\hline Anxiety subscale & & $7.41 \pm 4.18$ & $8.12 \pm 4.36$ & $7.77 \pm 4.28$ \\
\hline GSE & $\mathrm{M} \pm \mathrm{SD}$ & $30.73 \pm 4.98$ & $28.22 \pm 6.56$ & $29.44 \pm 5.97$ \\
\hline
\end{tabular}

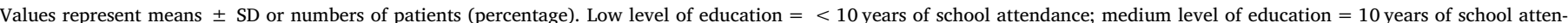

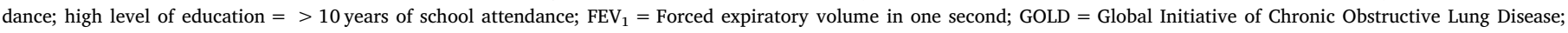

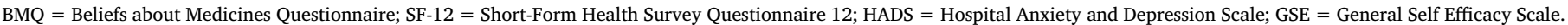

Table 2

MARS scores at different time points.

\begin{tabular}{llll}
\hline & Asthma & COPD & Total Sample \\
\hline Baseline (n) & 193 & 201 & 394 \\
Adherent n (\%)* & $36(18.7 \%)$ & $68(33.8 \%)$ & $104(26.4 \%)$ \\
M \pm SD & $21.2 \pm 3.7$ & $23.1 \pm 2.6$ & $22.2 \pm 3.3$ \\
Md [Q1;Q3] & $23.0[23 ; 25]$ & $24.0[23 ; 25]$ & $23.0[21 ; 25]$ \\
Follow-up after 3 months (n) & 110 & 142 & 252 \\
Adherent (n, \%)* & $16(14.5 \%)$ & $61(43 \%)$ & $77(30.6 \%)$ \\
M \pm SD & $20.9 \pm 3.6$ & $23.5 \pm 1.9$ & $22.4 \pm 3.0$ \\
Md [Q1;Q3] & $22.0[19 ; 24]$ & $24.0[23 ; 25]$ & $24.0[21 ; 25]$ \\
Follow-up after 1 year (n) & 73 & 98 & 171 \\
Adherent (n, \%)* & $13(17.8 \%)$ & $45(45.9 \%)$ & $58(33.9 \%)$ \\
M \pm SD & $21.6 \pm 3.3$ & $23.7 \pm 1.7$ & $22.8 \pm 2.7$ \\
Md [Q1;Q3] & $23.0[20 ; 24]$ & $24.0[23 ; 25]$ & $24.0[22 ; 25]$ \\
\hline
\end{tabular}

* Adherent behavior was defined by a MARS score of 25, MARS scores $<25$ indicated non-adherent behavior.

(Table 1). Patients who participated in the follow-up survey after one year had a significant higher Specific-necessity score $(M d n=4.2$, $Q 1 / Q 3=3.4 / 4.7)$ than patients who did not $(M d n=3.8$, $Q 1 / Q 3=3.2 / 4.55, U=22.195, p=0.009)$. For the other BMQ subscales no significant differences were identified.

\subsection{Associations between medication adherence and beliefs about medicines}

At baseline the association between general beliefs about harm and overuse were found to be significantly associated with a lower chance of being adherent for patients with asthma. Furthermore Specific-necessity showed a significant positive association with adherent behavior in both groups of patients. The detailed results of the baseline analysis have already been published [41].

Analysing the prospective associations, multivariable analyses showed that a higher score on the BMQ subscale General-overuse was associated with a lower chance of being adherent in patients with asthma at the follow-up after 3 months ( $\mathrm{OR}=0.76, p=0.483)$ and the follow-up after one year ( $\mathrm{OR}=0.42, p=0.094$ ). (For the results of the multivariable analyses see Tables 3 and 4). In patients with COPD General-overuse was associated with lower chances of adherence at the 3-month follow-up ( $\mathrm{OR}=0.77, p=0.328$ ) and the follow-up after one year ( $\mathrm{OR}=0.60, p=0.125)$. General-harm was associated with a lower chance of adherent behavior in patients with asthma at the first (OR $=0.75, p=0.549$ ) and the second follow-up $(\mathrm{OR}=0.33, p=0.084)$. For the patients with COPD an inverse association was found regarding General-harm and adherence at the 
Table 3

Unadjusted and adjusted logistic regression models of the association between the BMQ subscales and medication adherence in patients with asthma at follow-up.

\begin{tabular}{|c|c|c|c|c|c|c|c|}
\hline & & \multicolumn{3}{|c|}{ Follow-up after 3 months* } & \multicolumn{3}{|c|}{ Follow-up after one year** } \\
\hline & Model & $\mathrm{N}$ & OR $[95 \% \mathrm{CI}]$ & $\mathrm{p}$ & $\mathrm{N}$ & OR $[95 \% \mathrm{CI}]$ & $\mathrm{p}$ \\
\hline \multirow[t]{2}{*}{ BMQ-general-overuse } & adjusted & 109 & $0.76[0.34-1.66]$ & 0.483 & 71 & $0.42[0.15-1.16]$ & 0.094 \\
\hline & unadjusted & 109 & $0.69[0.34-1.43]$ & 0.319 & 72 & $0.42[0.17-1.00]$ & 0.049 \\
\hline \multirow[t]{2}{*}{ BMQ-general-harm } & adjusted & 110 & $0.75[0.28-1.95]$ & 0.549 & 72 & $0.33[0.10-1.16]$ & 0.084 \\
\hline & unadjusted & 110 & $0.64[0.26-1.56]$ & 0.324 & 73 & $0.40[0.14-1.12]$ & 0.081 \\
\hline \multirow[t]{2}{*}{ BMQ-general-utility } & adjusted & 109 & $1.27[0.49-3.28]$ & 0.628 & 72 & $1.70[0.50-5.75]$ & 0.397 \\
\hline & unadjusted & 109 & 1.17 [0.47-2.95] & 0.732 & 72 & $2.07[0.65-6.62]$ & 0.222 \\
\hline \multirow[t]{2}{*}{ BMQ-specific-necessity } & adjusted & 109 & $1.76[0.81-3.86]$ & 0.156 & 71 & $2.40[0.95-6.06]$ & 0.065 \\
\hline & unadjusted & 109 & $2.33[1.16-4.67]$ & 0.017 & 72 & $1.45[0.72-2.95]$ & 0.299 \\
\hline \multirow[t]{2}{*}{ BMQ-specific-concerns } & adjusted & 107 & $0.86[0.34-2.19]$ & 0.748 & 69 & $0.56[0.20-1.59]$ & 0.275 \\
\hline & unadjusted & 107 & 1.07 [0.44-2.57] & 0.887 & 70 & $0.59[0.23-1.51]$ & 0.267 \\
\hline
\end{tabular}

* adjusted for age, level of education, number of pills, number of other medications.

** adjusted for adults living in the same household, number of inhaler medications, distance to the physician, Asthma Control Questionnaire (ACQ).

follow-up after 3 months (OR $=0.91, p=0.738)$ and after one year (OR $=0.67, p=293$ ). Regarding the BMQ subscale General-utility, a higher score was associated with a higher chance of showing adherent behavior in both groups, except for the COPD patients at the follow-up after one year ( $\mathrm{OR}=0.93, p=0.850$ ). None of the associations between the General-beliefs subscales and adherence was found to be significant. Patients with a higher score in the BMQ Specific-necessity subscale had a higher chance of being adherent. In patients with asthma this association was neither found to be significant at the follow-up after three months $(\mathrm{OR}=1.76, p=0.156)$ nor at the follow-up after one year ( $\mathrm{OR}=2.4, p=0.065)$. At the three months follow-up higher scores on the Specific-necessity subscale significantly increased the chances of showing adherent behavior in patients with COPD (OR $=2.63, p=0.005$ ). An inverse association was found between the BMQ Specific-concerns subscale and adherence. For patients with COPD, this association was significant at three months ( $\mathrm{OR}=0.55, p=0.032)$ and after one year $(\mathrm{OR}=0.36, p=0.011)$. Testing for multicollinearity showed no strong relationships between the factors the models were adjusted for. Sensitivity analyses with two different MARS cut-off scores did not show substantially different associations regarding strength and significance (data not shown). Another sensitivity analysis which included participants of the one year follow-up only did also not show substantial deviations from the original models.

\section{Discussion}

At the baseline of our longitudinal study general beliefs about overuse and harm were significantly associated with a lower chance of adherent behavior in patients with asthma, while Specific-necessity was significantly associated with medication adherence in both groups of patients. The results of the analysis of the relationship between beliefs about medicines and medication adherence at baseline have been published elsewhere [41] while this paper focusses on the analyses of the longitudinal data.

Main findings of the longitudinal study were: The associations between the beliefs at baseline and adherence were consistent for both groups of patients at both follow-ups and showed the expected direction. However, a significant association could only be found regarding the Specific-necessity and the Specific-concerns subscales for the COPD sample. This could indicate a difference concerning the importance of the influence of medication related beliefs on adherence in both groups of patients. However, the comparison of the results of the two illness groups is limited by the different characteristics of the two population in our study.

For the COPD sample, in the follow-up after three months Specificnecessity had a significant positive association with adherence. This is in line with the findings obtained when analysing the association between beliefs about medicines and medication adherence at baseline. However, in the follow-up after one year this association remained positive but did not reach significance. This might be due to the small COPD sample of 86 patients that remained in the analysis. Patients that consider their medication as necessary to maintain their health status and prevent deterioration are more likely to take their medication as prescribed [1]. Our results are consistent with the findings of George et al. [26], who found that adherent patients had stronger beliefs in the necessity of their medication in a study with 276 COPD patients. On the other hand, in a study of 188 COPD patients, beliefs about the Specificnecessity were not an independent predictor of medication adherence [25]. Specific-concerns showed a significant inverse association with

Table 4

Unadjusted and adjusted logistic regression models of the association between the BMQ subscales and medication adherence in patients with COPD at follow-up.

\begin{tabular}{|c|c|c|c|c|c|c|c|}
\hline & & \multicolumn{3}{|c|}{ Follow-up after 3 months* } & \multicolumn{3}{|c|}{ Follow-up after one year** } \\
\hline & Model & $\mathrm{N}$ & OR $[95 \% \mathrm{CI}]$ & $\mathrm{p}$ & $\mathrm{N}$ & OR $[95 \% \mathrm{CI}]$ & $\mathrm{p}$ \\
\hline \multirow[t]{2}{*}{ BMQ-general-overuse } & adjusted & 129 & $0.77[0.46-1.30]$ & 0.328 & 85 & $0.60[0.31-1.15]$ & 0.125 \\
\hline & unadjusted & 140 & $0.75[0.46-1.20]$ & 0.228 & 96 & $0.66[0.37-1.16]$ & 0.146 \\
\hline \multirow[t]{2}{*}{ BMQ-general-harm } & adjusted & 122 & $0.91[0.51-1.61]$ & 0.738 & 80 & $0.67[0.31-1.42]$ & 0.293 \\
\hline & unadjusted & 132 & $0.73[0.43-1.24]$ & 0.246 & 91 & $0.59[0.30-1.17]$ & 0.131 \\
\hline \multirow[t]{2}{*}{ BMQ-general-utility } & adjusted & 126 & $1.16[0.69-1.97]$ & 0.570 & 83 & $0.93[0.43-2.02]$ & 0.850 \\
\hline & unadjusted & 137 & $1.17[0.72-1.90]$ & 0.534 & 94 & $1.38[0.72-2.65]$ & 0.331 \\
\hline \multirow[t]{2}{*}{ BMQ-specific-necessity } & adjusted & 130 & $2.63[1.35-5.12]$ & 0.005 & 86 & $2.01[0.91-4.45]$ & 0.085 \\
\hline & unadjusted & 141 & $1.55[0.94-2.55]$ & 0.086 & 97 & $2.05[1.07-3.92]$ & 0.030 \\
\hline \multirow[t]{2}{*}{ BMQ-specific-concerns } & adjusted & 125 & $0.55[0.32-0.95]$ & 0.032 & 84 & $0.36[0.17-0.79]$ & 0.011 \\
\hline & unadjusted & 136 & $0.46[0.28-0.77]$ & 0.003 & 94 & $0.33[0.17-0.66]$ & 0.002 \\
\hline
\end{tabular}

* adjusted for side effects, SF-12 Physical component summary, SF-12 Mental component summary.

** adjusted for age, number of inhaler medications, duration of disease (years), side effects, General Self Efficacy Scale (GSE). 
medication adherence in both follow-ups in our COPD sample. Remarkably, in the baseline analysis this inverse association had lost statistical significance after controlling for potential confounders. Our findings are supported by Krauskopf et al. [25] who found that higher scores on the Specific-concerns scales were associated with a lower odds of being adherent. In the study of Khdour et al. [12] adherence behavior was correlated with perceived barriers of medication (such as side effects) emphasizing the fact that Specific-concerns about medication influence the chance of being adherent. Another study in COPD patients showed that adherent patients had fewer concerns about their medication than patients that were non-adherent [26]. In contrast to our findings, a recently published prospective study over a 1-month-period showed no association between adherence measured using electronic monitoring and beliefs about medicines in a sample of patients with COPD recently discharged from a hospital [42].

While general beliefs about harm and overuse as well as Specificnecessity were significantly associated with adherence in patients with asthma at baseline none of the associations between beliefs and adherence was significant for these patients when analysing the longitudinal data but a consistent tendency over time was observed. In a longitudinal study performed in Australia beliefs about medicines were not identified as predictors of inhaler technique maintenance in patients with asthma [28]. Other previous studies showed a positive association between Specific-necessity and medication adherence in patients with asthma [23,24] as it was found in our study. The results of Sofianou et al. [13] support our findings showing that poor adherence was more common among patients with asthma who believed less in the necessity of their medication and were more concerned about their medication. Studies by Horne et al. showed a positive correlation between adherence and Specific-necessity as well as a negative correlation between adherence and Specific-concerns in patients with asthma $[15,16]$. Our results can be seen as consistent with the findings of these studies. A level of significance might have been reached with a larger number of participants.

The adherence level of $33 \%$ in our study is in line with some previous studies [11,26] but other studies found higher levels [12-14,24]. This might be explained by differing ways of measuring adherence and differences in the study populations.

Adherence was measured by self-report. Self-reports are often considered to be biased because of the social desirability and they tend to underestimate non-adherence by almost $20 \%$ [43]. However, it has also been shown that self-reported adherence correlates well with objectively measured adherence [43]. Furthermore, the questions of the MARS are expressed as negative statements to minimize social desirability [44]. In our study, the social desirability bias might have been a problem predominantly in the baseline survey where patients were recruited in a hospital or a practice. In the follow-ups, patients completed questionnaires at home and social expectations might have been less important. We did not collect any data that would provide external validation of our adherence data (e.g. pharmacy refill or electronic monitoring). Furthermore we did not collect information on reasons for loss to follow-up. This missing information is certainly a limitation of our study.

The majority of our patients with asthma was recruited in an outpatient setting. It is therefore difficult to generalize our results to all patients with asthma. Further research should include patients with more severe asthma. Only $6 \%$ of all COPD patients were classified as stage GOLD I and about $70 \%$ were inpatients. Therefore our results might not reflect the situation of patients with less severe COPD.

\section{Conclusions}

Our findings emphasize the importance of beliefs about medicines in relation to medication adherence in patients with COPD. The longitudinal study design allows a very cautious interpretation of the observed relationships in terms of causality. As the Specific-beliefs subscales were significantly associated with adherence, physicians might address primarily the concerns patients have about their own medication and strengthen their beliefs in the necessity of their own medicine to improve medication adherence.

By acknowledging patients' fears and beliefs about treatment particularly medication, non-adherence to medication can potentially be diminished, but randomised controlled trials are necessary to test tailored interventions. Future observational work should aim for greater numbers of patients at follow-up and include patients with a wider distribution of disease severity.

\section{Authors' contributions}

WF collected data for the study, entered data, analysed data, wrote the manuscript and reviewed it for important intellectual content. SB conceived and designed the study, interpreted the data, was responsible for quality control and reviewed the manuscript for important intellectual content. MB collected data for the study, entered data and reviewed the manuscript for important intellectual content. TF collected data for the study, entered data and reviewed the manuscript for important intellectual content. MMB analysed the data, was responsible for quality control and reviewed the manuscript for important intellectual content. MP contributed to the design of the study, helped interpret the clinical implications of the findings and reviewed the manuscript for important intellectual content. CA conceived and designed the study, interpreted the data, wrote the manuscript and reviewed it for important intellectual content. All authors have read and approved the final version of manuscript.

\section{Acknowledgements}

We would like to thank the physicians Frank Heinemann, Markus Reuter, Sigrid Karrer, Christof Kundel, Jürgen Pahnke, Wolfgang Sieber and Rupert Zach for facilitating recruitment of patients for this study. We thank Susanne Hammer, Florian Herl and Ursula Zach for their valuable help in patient recruitment and data entry. Finally, we are grateful to all patients who volunteered in this study.

\section{Funding}

The study was funded by internal funds of the Medical Sociology unit and the funds of the Faculty of Medicine of the University of Regensburg (ReForM-A-10-10-2013), earned by Susanne Brandstetter.

\section{Competing interests}

All authors have completed the ICMJE uniform disclosure form at www.icmje.org/coi_disclosure.pdf and declare: no support from any organization for the submitted work; no financial relationships with any organisations that might have an interest in the submitted work in the previous three years; no other relationships or activities that could appear to have influenced the submitted work. Prof. Dr. Apfelbacher reports non-financial support from Cogitando Healthcare Communication, outside the submitted work.

\section{Ethics approval}

The study was approved by the ethics committee of the University of Regensburg (file-numbers: 12-101-0162; 13-101-0091).

\section{Data sharing}

The data set is available from the authors upon reasonable request. 


\section{References}

[1] R. Horne, Representations of medication and treatment: advances in theory and measurement, in: K.J. Petrie, J.A. Weinman (Eds.), Perceptions of Health and Illness: Current Research and Applications, Harwood Academic Publishers, Amsterdam, 1997, pp. 155-188.

[2] J. Bourbeau, S.J. Bartlett, Patient adherence in COPD, Thorax 63 (9) (2008) 831-838.

[3] K. Sumino, M.D. Cabana, Medication adherence in asthma patients, Curr. Opin. Pulm. Med. 19 (1) (2013) 49-53.

[4] Global Initiative for Asthma (GINA), Global Strategy for Asthma Management and Prevention (Updated), www.ginasthma.org, (2016), Accessed date: December 2016.

[5] Global Initiative for Chronic Obstructive Lung Disease, Global Strategy for the Diagnosis, Management, and Prevention of Chronic Obstructive Pulmonary Disease (Updated), www.goldcopd.org/global-strategy-diagnosis-management-preventioncopd-2016/, (2016) , Accessed date: April 2017.

[6] G.J. Gibson, R. Loddenkemper, Y. Sibille, et al., The European Lung White Book: Respiratory Health and Disease in Europe, Sheffield, Charlesworth Press, 2013, pp. $16-27$.

[7] A. Raadts, U. Juergens, Volkswirtschaftliche Bedeutung von Asthma und COPD, in: H. Lingner, F.W. Schwartz, K. Schultz (Eds.), Volkskrankheit Asthma/COPD, Springer, Heidelberg, 2007, pp. 37-48.

[8] E.D. Bateman, H.A. Boushey, J. Bousquet, et al., Can guideline-defined asthma control be achieved? The gaining optimal asthma control study, Am. J. Respir. Crit. Care Med. 170 (8) (2004) 836-844.

[9] P. Calverley, R. Pauwels, J. Vestbo, et al., Combined salmeterol and fluticasone in the treatment of chronic obstructive pulmonary disease: a randomized controlled trial, Lancet 361 (9356) (2003) 449-456.

[10] S. Suissa, P. Ernst, A. Kezouh, Regular use of inhaled corticosteroids and the long term prevention of hospitalisation for asthma, Thorax 57 (10) (2002) 880-884.

[11] E. Mehuys, K. Boussery, E. Adriaens, et al., COPD management in primary care: an observational, community pharmacy-based study, Ann. Pharmacother. 44 (2) (2010) 257-266.

[12] M.R. Khdour, A.F. Hawwa, J.C. Kidney, et al., Potential risk factors for medication non-adherence in patients with chronic obstructive pulmonary disease (COPD), Eur. J. Clin. Pharmacol. 68 (10) (2012) 1365-1373.

[13] A. Sofianou, M. Martynenko, M.S. Wolf, et al., Asthma beliefs are associated with medication adherence in older asthmatics, J. Gen. Intern. Med. 28 (1) (2013) $67-73$.

[14] T.T. Menckeberg, M.L. Bouvy, M. Bracke, et al., Beliefs about medicines predict refill adherence to inhaled corticosteroids, J. Psychosom. Res. 64 (1) (2008) 47-54.

[15] R. Horne, J. Weinman, Patients' beliefs about prescribed medicines and their role in adherence to treatment in chronic physical illness, J. Psychosom. Res. 47 (6) (1999) $555-567$.

[16] R. Horne, J. Weinman, Self-regulation and self-management in asthma: exploring the role of illness perceptions and treatment beliefs in explaining non-adherence to preventer medication, Psychol. Health 17 (1) (2002) 17-32.

[17] M.A. Diefenbach, H. Leventhal, The common-sense model of illness representation: theoretical and practical considerations, J. Soc. Distress Homeless 5 (1) (1996) $11-38$.

[18] R. Horne, S.C.E. Chapman, R. Parham, et al., Understanding Patients' adherencerelated beliefs about medicines prescribed for long-term conditions: a meta-analytic review of the necessity-concerns framework, PLoS One 8 (12) (2013) e80633.

[19] Y. Nestoriuc, J.A. Glombiewski, R. Horne, et al., Beeinflusst die Einstellung zu Medikamenten deren Einnahme? Validierung des "Beliefs about Medicines" Fragebogens in der Allgemeinbevölkerung, Z. Klin. Psychol. Psychother. 39 (2010) 64-69.

[20] R. Horne, J. Weinman, M. Hankins, The beliefs about medicines questionnaire: the development and evaluation of a new method for assessing the cognitive representation of medication, Psychol. Health 14 (1) (1999) 1-24.

[21] C. Mahler, K. Hermann, R. Horne, et al., Patients' beliefs about medicines in a primary care setting in Germany, J. Eval. Clin. Pract. 18 (2) (2012) 409-413.

[22] H.M. Phatak, J. Thomas, Relationships between beliefs about medications and nonadherence to prescribed chronic medications, Ann. Pharmacother. 40 (10)
(2006) 1737-1742.

[23] M. Emilsson, I. Berndtsson, J. Lotvall, et al., The influence of personality traits and beliefs about medicines on adherence to asthma treatment, Prim. Care Respir. J. 20 (2) (2011) 141-147.

[24] M. van Steenis, J. Driesenaar, J. Bensing, et al., Relationship between medication beliefs, self-reported and refill adherence, and symptoms in patients with asthma using inhaled corticosteroids, Patient Prefer Adherence 8 (2014) 83-91.

[25] K. Krauskopf, A.D. Federman, M.S. Kale, et al., Chronic obstructive pulmonary disease illness and medication beliefs are associated with medication adherence, COPD 12 (2) (2015) 151-164.

[26] J. George, D.C.M. Kong, R. Thoman, et al., Factors associated with medication nonadherence in patients with COPD, Chest 128 (5) (2005) 3198-3204.

[27] Adherence to Long-Term Therapies: Evidence for Action. World Health Organization, www.who.int/chp/knowledge/publications/adherence_full_report. pdf, (2003), Accessed date: April 2017.

[28] L. Ovchinikova, L. Smith, S. Bosnic-Anticevich, Inhaler technique maintenance: gaining an understanding from the patient's perspective, J. Asthma 48 (6) (2011) 616-624

[29] C. Mahler, K. Hermann, R. Horne, et al., Assessing reported adherence to pharmacological treatment recommendations. Translation and evaluation of the medication adherence report scale (MARS) in Germany, J. Eval. Clin. Pract. 16 (3) (2010) 574-579.

[30] U. Opitz, M. Glattacker, J. Bengel, et al., Der "Beliefs about Medicines Questionnaire" (BMQ (C R Horne) - Übersetzung und erste methodische Prüfung an Patienten mit Fibromyalgie, in: Deutsche Rentenversicherung Bund (Ed.), Internetausgabe des Tagungsbandes zum 17. Rehabilitationswissenschaftlichen Kolloquium, 2008 www.eservice-drv.de/ForschPortalWeb/ressource?key= tagungsband_17_reha_kolloqu.pdf\#page $=100$, Accessed date: April 2017.

[31] P.W. Jones, G. Harding, P. Berry, et al., Development and first validation of the COPD assessment test, Eur. Respir. J. 34 (3) (2009) 648-654.

[32] E.F. Juniper, O'byrne PM, G. Guyatt, et al., Development and validation of questionnaire to measure asthma control, Eur. Respir. J. 14 (4) (1999) 902.

[33] N. Gupta, L.M. Pinto, A. Morogan, et al., The COPD assessment test: a systematic review, Eur. Respir. J. 44 (4) (2014) 873-884.

[34] COPD, Assessment Test Healthcare Professional User Guide: Expert Guidance on frequently Asked Questions, www.catestonline.org/images/UserGuides/CAT_HCP \%20User\%20Guide.pdf , Accessed date: April 2017.

[35] E.F. Juniper, K. Svensson, A.C. Mork, et al., Measurement properties and interpretation of three shortened versions of the asthma control questionnaire, Respir. Med. 99 (5) (2005) 553-558.

[36] B. Gandek, J.E. Ware, N.K. Aaronson, et al., Cross-validation of item selection and scoring for the SF-12 health survey in nine countries: results from the IQOLA project, J. Clin. Epidemiol. 51 (11) (1998) 1171-1178.

[37] J.E. Ware, M. Kosinski, S.D. Keller, A 12-item short-form health survey: construction of scales and preliminary tests of reliability and validity, Med. Care 34 (3) (1996) 220-233.

[38] A. Luszczynska, U. Scholz, R. Schwarzer, The general self-efficacy scale: multicultural validation studies, Aust. J. Psychol. 139 (5) (2005) 439-457.

[39] A.S. Zigmond, R.P. Snaith, The hospital anxiety and depression scale, Acta Psychiatr. Scand. 67 (6) (1983) 361-370.

[40] I. Bjelland, A.A. Dahl, T.T. Haug, et al., The validity of the hospital anxiety and depression scale an updated literature review, J. Psychosom. Res. 52 (2002) 69-77.

[41] S. Brandstetter, T. Finger, W. Fischer, et al., Differences in medication adherence are associated with beliefs about medicines in asthma and COPD, Clin. Transl. Allergy 7 (2017) 39.

[42] I. Sulaiman, B. Cushen, G. Greene, et al., Objective assessment of adherence to inhalers by patients with chronic obstructive pulmonary disease, Am. J. Respir. Crit. Care Med. 195 (10) (2017) 1333-1343.

[43] R.B. Haynes, D. Taylor, D. Sackett, et al., Can simple clinical measurements detect patient noncompliance? Hypertension 2 (6) (1980) 757-764.

[44] J.L. Cohen, D.M. Mann, J.P. Wisnivesky, et al., Assessing the validity of self-reported medication adherence among inner-city asthmatic adults: the medication adherence report scale for asthma, Ann Allergy Asthma Immunol 103 (4) (2009) 325-331. 